Homology, Homotopy and Applications, vol.10(3), 2008, pp.223-268

\title{
THE SECOND REAL JOHNSON-WILSON THEORY AND NONIMMERSIONS OF $R P^{n}$
}

\author{
NITU KITCHLOO AND W. STEPHEN WILSON
}

(communicated by Donald M. Davis)

\begin{abstract}
$\mathrm{Hu}$ and Kriz construct the real Johnson-Wilson spectrum, $E R(n)$, which is $2^{n+2}\left(2^{n}-1\right)$-periodic, from the $2\left(2^{n}-1\right)$ periodic spectrum $E(n) . E R(1)$ is just $K O_{(2)}$ and $E(1)$ is just $K U_{(2)}$. We compute $E R(n)^{*}\left(R P^{\infty}\right)$ and set up a Bockstein spectral sequence to compute $E R(n)^{*}(-)$ from $E(n)^{*}(-)$. We combine these to compute $E R(2)^{*}\left(R P^{2 n}\right)$ and use this to get new nonimmersions for real projective spaces. Our lowest dimensional new example is an improvement of 2 for $R P^{48}$.
\end{abstract}

\section{Introduction}

We have three main goals in this paper. First, we want to introduce the second real Johnson-Wilson cohomology, $E R(2)^{*}(-)$, as a real problem-solving tool. Second, we develop computational tools for $E R(2)^{*}(-)$ and third, we apply $E R(2)^{*}(-)$ and our computational tools to prove some new families of nonimmersions for real projective spaces. The lowest dimensional example is $R P^{48}$.

Our concern is with the real Johnson-Wilson cohomology, $E R(n)^{*}(X)$, developed in $[\mathbf{H K 0 1}]$, [Hu01], [KW07b], and [KW07a]. It is $2^{n+2}\left(2^{n}-1\right)$-periodic and constructed from the $2\left(2^{n}-1\right)$-periodic spectrum $E(n) . E R(1)$ is just $K O_{(2)}$ and $E(1)$ is just $K U_{(2)}$. Our long term goals are to develop and apply $E R(2)^{*}(-)$ but much of our preliminary work stands for $E R(n)^{*}(-)$ in general.

The coefficient ring, $E(n)^{*}\left(S^{0}\right)$, is given by $\mathbb{Z}_{(2)}\left[v_{1}, v_{2}, \ldots, v_{n}^{ \pm 1}\right]$ where the degree of $v_{k}$ is $-2\left(2^{k}-1\right)$. The theory, $E(n)$, is complex orientable and as such it has a formal group law with coefficients made up from the $v_{k}$ 's and a [2]-series,

$$
[2](u)=\sum_{k \geqslant 0} a_{k} u^{k+1}=\sum_{k \geqslant 0}^{n} F v_{k} u^{2^{k}} .
$$

We prefer to grade our cohomologies over $\mathbb{Z} /\left(2^{n+2}\left(2^{n}-1\right)\right)$. For $E(n)^{*}(-)$ we just set $v_{n}^{2^{n+1}}=1$.

We start off with a theorem, proven later as Theorem 3.2, that goes back to the equivariant roots of $E R(n)$.

The first author was supported by an NSF grant.

Received August 20, 2007, revised April 7, 2008; published on December 5, 2008.

2000 Mathematics Subject Classification: 55N20, 55N91, 55T25, 57R42.

Key words and phrases: real projective space, nonimmersions, Johnson-Wilson theories.

This article is available at http://intlpress.com/HHA/v10/n3/a11

Copyright (c) 2008, International Press. Permission to copy for private use granted. 
Theorem 1.2. Let $\lambda(n)=2^{2 n+1}-2^{n+2}+1$. Then there is a $u \in E R(n)^{1-\lambda(n)}\left(R P^{\infty}\right)$ and

$$
E R(n)^{*}\left(R P^{\infty}\right) \simeq E R(n)^{*}[[u]] /([2](u))
$$

where the $v_{k}$ are replaced by $v_{k}^{E R(n)} \in E R(n)^{(\lambda(n)-1)\left(2^{k}-1\right)}\left(S^{0}\right)$.

In our special case of interest, $E R(2), v_{2}^{E R(2)}=1$ and we rename $v_{1}^{E R(2)}$ as $\alpha$. Our relation becomes

$$
[2](u)=2 u+{ }_{F} \alpha u^{2}+{ }_{F} u^{4}
$$

and it maps to the same relation in $E(2)^{*}\left(R P^{\infty}\right)$ for our 48-periodic $E(2)$. To do this we have to replace the usual $x_{2} \in E(2)^{2}\left(R P^{\infty}\right)$ with the image of the $u \in$ $E R(2)^{-16}\left(R P^{\infty}\right)$, which is $v_{2}^{3} x_{2}$, and replace $v_{1}$ with the image of $\alpha \in E R(2)^{16}, v_{2}^{5} v_{1}$. Since $v_{2}$ is a unit, this is not a problem.

For our applications we need $E R(2)^{*}\left(R P^{2 n}\right)$, and the equivariant approach doesn't work here. The stable cofibration of $[$ KW07a],

$$
\Sigma^{\lambda(n)} E R(n) \stackrel{x}{\longrightarrow} E R(n) \longrightarrow E(n)
$$

gives us a long exact sequence:

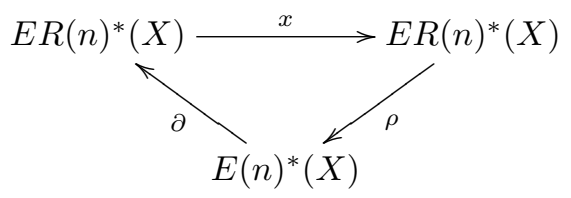

where $x$ lowers degree by $\lambda(n)$ and $\partial$ raises degree by $\lambda(n)+1$. This is a classic exact couple and leads us directly to a Bockstein spectral sequence for $x$-torsion. We know that $x^{2^{n+1}-1}=0$ so there can be only $2^{n+1}-1$ differentials. We set up this spectral sequence and compute $d^{1}$. In the case $n=1$ it can be used to compute $K O_{(2)}^{*}(X)$ from $K U_{(2)}^{*}(X)$. In this case there are only 3 differentials. For the case of interest to us, $n=2$, there are only 7 differentials and because for many of our spaces our $E^{1}$-term, $E(2)^{*}(X)$, is even degree, we have only 4 differentials because the $d^{2 r}$ are odd degree.

We use the Bockstein spectral sequence to compute $E R(2)^{*}\left(R P^{2 n}\right)$ after setting up the spectral sequence. This breaks up into 8 cases depending on $n$ modulo 8 . The descriptions can get lengthy (times 8) but can be read off directly from the Bockstein spectral sequence which is quite compact. Here we will be content to describe the part of $E R(2)^{*}\left(R P^{2 n}\right)$ we are really interested in, namely $E R(2)^{16 *}\left(R P^{2 n}\right)$.

We like to describe our groups, which are all modules over the 2 -adics, with what we call a 2 -adic basis, i.e. a set of elements such that any element in our group can be written uniquely as a finite sum of these elements with coefficients 0 or 1 . We tend to abuse the language by referring to a group as "represented by" or "given by" when we mean "has a 2-adic basis". If the group is infinite, it will have a topology on it and we can use unique infinite sums. Usually we can compute 2 times an element by using (1.3).

Keep in mind that, using this notation, the usual $E(2)^{*}\left(R P^{2 n}\right)$ has a 2-adic basis given by $\alpha^{k} u^{j}$, with $0 \leqslant k$ and $0<j \leqslant n$ and the 48 -periodic version has basis given 
by $v_{2}^{i} \alpha^{k} u^{j}$, with $0 \leqslant i<8$. We always use reduced cohomology.

A simplified version of Theorem 13.4 is given by:

Theorem 1.6. A 2-adic basis for $E R(2)^{16 *}\left(R P^{2 n}\right)$ consists of the elements $\alpha^{k} u^{j}$, with $0 \leqslant k$ and $0<j \leqslant n$, and when

$n \equiv 0$ or 7 modulo $8, u^{n+1}=0$;

$n \equiv 1$ or 6 modulo $8, \alpha^{k} u^{n+1}$, with $u^{n+2}=0$;

$n \equiv 2$ or 5 modulo $8, \alpha^{k} u^{n+1}$, and $u^{n+2}$, with $u^{n+3}=0$;

$n \equiv 3$ or 4 modulo $8, u^{n+1}, u^{n+2}$, and $u^{n+3}$, with $u^{n+4}=0$;

and no others.

Our applications use the four cases, $n \equiv 1,2,5$ and 6 , modulo 8 , where we have $\alpha^{k} u^{n+1}$. Here we have a purely algebraic, no topology implied or used, surjection

$$
E R(2)^{16 *}\left(R P^{2 n}\right) \longrightarrow E(2)^{16 *}\left(R P^{2 n+2}\right), \quad n \equiv 1,2,5,6 \bmod 8 .
$$

This was the key to inspiring our nonimmersion results. We also need the isomorphism given above for

$$
E R(2)^{16 *}\left(R P^{2 n}\right) \longrightarrow E(2)^{16 *}\left(R P^{2 n}\right), \quad n \equiv 0,7 \bmod 8 .
$$

From [Jam63] we know that if there is an immersion of $R P^{2 n}$ into $\mathbb{R}^{2 k}$ then there is an axial map:

$$
R P^{2 n} \times R P^{2^{K}-2 k-2} \longrightarrow R P^{2^{K}-2 n-2} .
$$

Don Davis, in [Dav84], shows that there is no such map when

$$
n=m+\alpha(m)-1 \text { and } k=2 m-\alpha(m),
$$

where $\alpha(m)$ is the number of ones in the binary expansion of $m$. He does this by showing that the image of $u^{2^{K-1}-n}$ on the right side of

$$
E(2)^{*}\left(R P^{2 n}\right) \otimes_{E(2)^{*}} E(2)^{*}\left(R P^{2^{K}-2 k-2}\right) \longleftarrow E(2)^{*}\left(R P^{2^{K}-2 n-2}\right)
$$

is nonzero on the left. However, this power of $u$ on the right is zero. He further needs that the tensor product injects to $E(2)^{*}\left(R P^{2 n} \times R P^{2^{K}-2 k-2}\right)$.

This computation is actually a coproduct because it can first be carried out for the map $R P^{\infty} \leftarrow R P^{\infty} \times R P^{\infty}$ and this has a Künneth isomorphism for both our theories $E R(2)^{*}(-)$ and $E(2)^{*}(-)$. The first step,

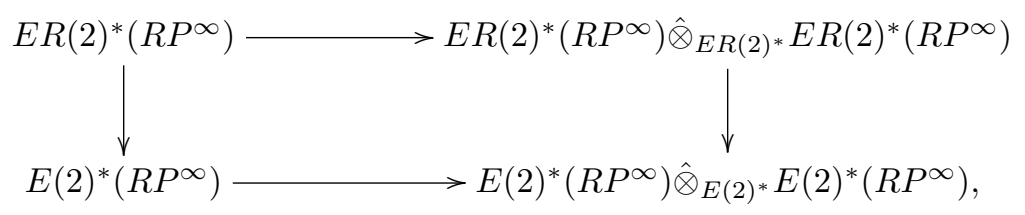

is an isomorphism from the top row to the bottom in degrees $16 *$ by Corollaries 8.3 and 17.3. Don Davis shows that the image of $u^{2^{K-1}-n}$ maps nontrivially to $E(2)^{16 *}\left(R P^{2 n} \times R P^{2^{K}-2 k-2}\right)$. The obstruction can be written in terms of the 2adic basis for this. We show that the same elements exist and are basis elements for $E R(2)^{16 *}\left(R P^{2 n} \times R P^{2^{K}-2 k-4}\right)$ and so the obstruction must also be nonzero here. 
This argument and computation gives us an improvement of 2 in our nonimmersions over previously known. The abelian group structure of the tensor product is extremely complicated but by relying on [Dav84] we avoid ever having to consider it.

We need to restrict our attention to the cases $n \equiv 0$ and 7 modulo 8 and $-k-2$ equal to $1,2,5$ or 6 modulo 8 in order to get our results. Our nonimmersion theorem is:

Theorem 1.9. When the pair $(m, \alpha(m))$ is, modulo 8, $(2,7),(7,2),(6,3),(3,6)$, $(7,1),(4,4),(3,5)$, or $(0,0)$, then

$$
R P^{2(m+\alpha(m)-1)} \text { does not immerse }(\not) \text { in } \mathbb{R}^{2(2 m-\alpha(m)+1)} \text {. }
$$

When the pair $(m, \alpha(m))$ is, modulo $8,(4,3),(1,6),(0,7)$, or $(5,2)$, then

$$
R P^{2(m+\alpha(m))} \nsubseteq \mathbb{R}^{2(2 m-\alpha(m)+1)} \text {. }
$$

Although we don't have to deal with the abelian group structure that makes Don Davis's work so difficult, this theorem is not for free from his work. By looking at the $\operatorname{ER}(2)^{*}$ submodules of our groups generated by elements of degree $16 *$ it is fairly easy to show that our obstruction is nontrivial in the tensor product. The injection of the tensor product,

$$
E(2)^{*}\left(R P^{2 m}\right) \otimes_{E(2)^{*}} E(2)^{*}\left(R P^{2 n}\right) \longrightarrow E(2)^{*}\left(R P^{2 m} \wedge R P^{2 n}\right),
$$

is ancient knowledge. We don't have such a result for $E R(2)^{*}(-)$. Where much of our work comes in is to show that the relevant basis elements map independently to $E R(2)^{*}(-)$ of the product. We do not have to compute the entirety of the $E R(2)$ cohomology of the product but just enough to give us what we need.

Looking closely at our theorem to decide if we really have anything new or not, let's take the pair $(m, \alpha(m))=(6,3)$. Let $m=2+4+2^{i}, i>3$, then $2 n=2(m+\alpha(m)-$ $1)=2\left(2+4+2^{i}+3-1\right)=16+2^{i+1}$ and $2(2 m-\alpha(m)+1)=2\left(4+8+2^{i+1}-3+\right.$ $1)=4+16+2^{i+2}=20+2^{i+2}$. Our result, in this case, shows that $R P^{16+2^{i+1}} \nsubseteq$ $\mathbb{R}^{20+2^{i+2}}$. Looking at the best known results, compiled by Don Davis in [Dav], we know that $R P^{16+2^{i+1}} \nsubseteq \mathbb{R}^{18+2^{i+2}}$ but $R P^{16+2^{i+1}} \subseteq \mathbb{R}^{23+2^{i+2}}$. Furthermore, when $i=4,5$ and 6 we get results for very low spaces:

$$
R P^{48} \nsubseteq \mathbb{R}^{84} ; \quad R P^{80} \nsubseteq \mathbb{R}^{148} ; \quad R P^{144} \nsubseteq \mathbb{R}^{276} .
$$

Our claim is that this alone makes a good case for $E R(2)^{*}(-)$ as a powerful tool. There are only 8 projective spaces, $R P^{n}$, with $n \leqslant 50$, where the best possible results are not yet known. For these 8 spaces there were a total of 26 gaps, now 24 . Our result is the first improvement in over 20 years for any $R P^{n}$ with $n \leqslant 50$.

The pair $(4,4)$ gives

$$
R P^{62+2^{i}} \nsubseteq \mathbb{R}^{106+2^{i+1}}
$$

where $i>5$. The lowest cases here are

$$
R P^{126} \nsubseteq \mathbb{R}^{234} \quad R P^{190} \nsubseteq \mathbb{R}^{362}
$$

These are nice because they get onto Don Davis's tables, [Dav], just barely, but 
at least this way we know we have something new, which would be difficult to tell otherwise.

From the second part of the theorem we only get an improvement of one dimension. The pair $(4,3)$ gives $m=4+2^{i}+2^{j}$ with $2<i<j$. We get

$$
R P^{14+2^{i+1}+2^{j+1}} \nsubseteq \mathbb{R}^{12+2^{i+2}+2^{j+2}}
$$

With $i=3$ we get

$$
R P^{30+2^{j+1}} \nsubseteq \mathbb{R}^{44+2^{j+2}}
$$

with lowest examples

$$
R P^{62} \nsubseteq \mathbb{R}^{108} ; \quad R P^{94} \nsubseteq \mathbb{R}^{172} ; \quad R P^{158} \nsubseteq \mathbb{R}^{300}
$$

With $i=4$ we get

$$
R P^{46+2^{j+1}} \nsubseteq \mathbb{R}^{76+2^{j+2}}
$$

with lowest examples

$$
R P^{110} \nsubseteq \mathbb{R}^{204} ; \quad R P^{174} \nsubseteq \mathbb{R}^{332} .
$$

For the $i=3$ and 4 cases above, there is now just a gap of 1 between known nonimmersions and immersions.

Don Davis, [Dav], keeps track of the best results for $R P^{d+2^{i}}$ for $0 \leqslant d<64$. Of these 64,24 are best possible at this time. We have improved the results for 4 of the 40 remaining: $d=16,30,46$ and 62 . We conjecture that this machine can improve results for $d=32,48,49,54,56$ and 57 , but these could be computationally intensive and so don't fit into this paper.

There is a bit of a saga associated with the $R P^{48}$ case. The theory tmf is clearly stronger than $E R(2)$ so the question arose as to why [BDM02] didn't see this result. When they looked again at their results, they realized that they had actually stated a family that included this case but had overlooked it when converting to the tables [Dav]. A closer look at [BDM02] revealed a simplification that had not been justified. That allows us to technically slip in with this result before they managed to patch up some of their theorems, which now include this. The theory tmf is very complicated and because of this complexity it cannot approach our other results at present.

The paper begins by computing $E R(n)^{*}\left(R P^{\infty}\right)$ using the equivariant approach. We then set up the Bockstein spectral sequence for computing $E R(n)^{*}(-)$ from $E(n)^{*}(-)$. We use the Bockstein spectral sequence to compute all of the (eight) cases of $E R(2)^{*}\left(R P^{2 n}\right)$. When this is done, we extract, from the Bockstein spectral sequence, just what we need about $E R(2)^{*}(-)$ of products. Then we wrap things up by producing our nonimmersion results.

The authors had worked with $E R(n)$ with an emphasis on $E R(2)$ before with an eye to eventually attacking nonimmersion problems. This project really got underway at the Bendersky-Davis 60th birthday conference at Newark, Delaware, April 2005, where, over lunch, the second author was inspired to work on the problem by Jesus González and Martin Bendersky who have continued to correspond with the authors throughout the project. Don Davis then joined the group, and without his help and 
tables we would never even know if we had new results. Thanks to all three. Special thanks are also in order for the referee's careful reading and numerous helpful comments.

At the end of the paper we have a short section explaining how to compute $E R(2)^{*}\left(R P^{2 n}\right)$ using the Atiyah-Hirzebruch spectral sequence. This was how it was first done and it required some interesting twists. Then it was thought that the Bockstein spectral sequence approach was unworkable. The Atiyah-Hirzebruch spectral sequence approach broke down when it came to studying the products; things just got too complicated. We then resurrected the Bockstein spectral sequence approach which proved successful. One of the unexpected and unnecessarily complicating factors was our choice of product spaces to study first to learn about products. The case $R P^{16}$ was essential for the study of $E R(2)^{*}\left(R P^{2 n}\right)$ for the Atiyah-Hirzebruch spectral sequence approach and it is "nicer" than other $R P^{2 n}$. So $R P^{16} \times R P^{16}$ was chosen on the grounds that it should be both elementary and educational. It turns out that great simplification occurs when one space is bigger than the other, or, phrased differently, complications occurred for $R P^{16} \times R P^{16}$ that only occur when the spaces are the same. Much time was lost on these irrelevant complications.

\section{Equivariant results}

Recall from $[\mathbf{H K 0 1}]$ that there is a real spectrum $\mathbb{E}(n)$ corresponding to the Johnson-Wilson spectra. In particular, $\mathbb{E}(n)$ consists of a bigraded family of $\mathbb{Z} /(2)$ spaces $\mathbb{E}(n)_{(a, b)}$. We denote by $\mathbb{E} \mathbb{R}(n)_{(a, b)}$ the homotopy fixed point space of the $\mathbb{Z} /(2)$ action on $\mathbb{E}(n)_{(a, b)}$. The collection of spaces $\mathbb{E}(n)_{(k, 0)}$ form a (naive) $\mathbb{Z} /(2)$-equivariant omega spectrum, and we define the spectrum $E R(n)$ as the corresponding homotopy fixed point spectrum $\mathbb{E} R(n)_{(k, 0)}$. Furthermore, it is shown in [HK01] that the real spectrum $\mathbb{E}(n)$ satisfies a strong completion theorem, in the sense that the canonical map

$$
\iota: \mathbb{E}(n)_{(a, b)} \longrightarrow \operatorname{Map}\left(\mathbb{E} \mathbb{Z} /(2)_{+}, \mathbb{E}(n)_{(a, b)}\right)
$$

is a $\mathbb{Z} /(2)$-equivalence, where $\mathbb{E} \mathbb{Z} /(2)$ represents the free, contractible $\mathbb{Z} /(2)$-complex. For a space $X$ with a $\mathbb{Z} /(2)$-action, we may define bigraded cohomology groups $\mathbb{E} \mathbb{R}(n)^{a, b}(X)[\mathbf{H K 0 1}]$ as the groups

$$
\mathbb{E} \mathbb{R}(n)^{(a, b)}(X)=\pi_{0} \operatorname{Map}^{\mathbb{Z} /(2)}\left(X, \mathbb{E}(n)_{(a, b)}\right) .
$$

The strong completion theorem has a few useful consequences:

Proposition 2.1. Let $X$ be a pointed space with the trivial $\mathbb{Z} /(2)$ action. Then the map $\iota$ above induces an isomorphism:

$$
\mathbb{E} \mathbb{R}(n)^{(k, 0)}(X) \longrightarrow E R(n)^{k}(X) .
$$

The proof of the above proposition follows directly from the strong completion theorem, and is left to the reader. Another useful consequence of the strong completion theorem is the following:

Proposition 2.2. Let $X$ and $Y$ be pointed $\mathbb{Z} /(2)$-spaces. Assume that $f: X \rightarrow Y$ is $a \mathbb{Z} /(2)$-equivariant map that is a homotopy equivalence (non equivariantly). Then $f$ 
induces an isomorphism:

$$
f^{*}: \mathbb{E} \mathbb{R}(n)^{(a, b)}(Y) \longrightarrow \mathbb{E} \mathbb{R}(n)^{(a, b)}(X) .
$$

Proof. By the strong completion theorem, we may write the groups $\mathbb{E} \mathbb{R}(n)^{(a, b)}(Z)$ as $\pi_{0} \operatorname{Map}^{\mathbb{Z} /(2)}\left(\mathbb{E} \mathbb{Z} /(2)_{+} \wedge Z, \mathbb{E}(n)_{(a, b)}\right)$, for an arbitrary $\mathbb{Z} /(2)$-space $Z$. Now consider the map

$$
I d \wedge f: \mathbb{E} \mathbb{Z} /(2)_{+} \wedge X \longrightarrow \mathbb{E} \mathbb{Z} /(2)_{+} \wedge Y .
$$

Since the spaces $\mathbb{E} \mathbb{Z} /(2)_{+} \wedge X$ and $\mathbb{E} \mathbb{Z} /(2)_{+} \wedge Y$ are free $\mathbb{Z} /(2)$-spaces, the map $I d \wedge f$ is a $\mathbb{Z} /(2)$-homotopy equivalence. It follows from the above previous observation that $f^{*}$ is an isomorphism.

\section{Cohomology of projective spaces}

We shall use the above propositions to describe the $E R(n)$ cohomology of the infinite projective space. To this end, we need to consider the complex projective space $C P^{\infty}$, with the action of $\mathbb{Z} /(2)$ given by complex conjugation. The space $C P^{\infty}$ supports the $\mathbb{Z} /(2)$-equivariant tautological complex line bundle $\gamma$. Moreover, $\gamma$ is real-oriented, in the sense that it admits a real Thom class $t \in \mathbb{E} \mathbb{R}(n)^{(1,1)}(T h(\gamma))$. Let $u \in \mathbb{E} \mathbb{R}(n)^{(1,1)}\left(C P^{\infty}\right)$ denote the Euler class of $\gamma$. The standard argument using the Atiyah-Hirzebruch spectral sequence may be invoked in the real setting to show that $\mathbb{E} \mathbb{R}(n)^{(*, *)}\left(C P^{\infty}\right) \simeq \mathbb{E} \mathbb{R}(n)^{(*, *)}[[u]]$, as a bigraded ring [HK01].

Now consider the real bundle $\gamma^{\otimes 2}$. The Euler class of $\gamma^{\otimes 2}$ is simply [2](u). Let $R \tilde{P}^{\infty}$ denote the unit sphere bundle of $\gamma^{\otimes 2}$. Notice that $R \tilde{P}^{\infty}$ may be identified with the space of (real) lines in $\mathbb{C}^{\infty}$ and as such, it supports a nontrivial $\mathbb{Z} /(2)$-action given by complex conjugation. Let $f: R P^{\infty} \rightarrow R \tilde{P}^{\infty}$ denote the inclusion induced by the $\mathbb{R}^{\infty} \subset \mathbb{C}^{\infty}$. Notice that $f$ is a $\mathbb{Z} /(2)$-equivariant map with $R P^{\infty}$ having a trivial $\mathbb{Z} /(2)$-action. Moreover $f$ is a (non equivariant) homotopy equivalence. It follows from the previous proposition that:

Lemma 3.1. The map $f: R P^{\infty} \rightarrow R \tilde{P}^{\infty}$ induces an isomorphism

$$
f^{*}: \mathbb{E} \mathbb{R}(n)^{(a, b)}\left(R \tilde{P}^{\infty}\right) \longrightarrow \mathbb{E} \mathbb{R}(n)^{(a, b)}\left(R P^{\infty}\right) .
$$

We may calculate the cohomology of $R \tilde{P}^{\infty}$ using the Gysin sequence, [LMS86, Chapter X, Section 5] for the bundle $\gamma^{\otimes 2}$ :

$$
\begin{gathered}
\cdots \longrightarrow \mathbb{E} \mathbb{R}(n)^{(a-1, b-1)}\left(C P^{\infty}\right) \stackrel{[2](u)}{\longrightarrow} \mathbb{E} \mathbb{R}(n)^{(a, b)}\left(C P^{\infty}\right) \longrightarrow \\
\mathbb{E} \mathbb{R}(n)^{(a, b)}\left(R \tilde{P}^{\infty}\right) \longrightarrow \mathbb{E} \mathbb{R}(n)^{(a, b-1)}\left(C P^{\infty}\right) \longrightarrow \cdots .
\end{gathered}
$$

Since $[2](u)$ is clearly not a zero divisor in $\mathbb{E} \mathbb{R}(n)^{(*, *)}\left(C P^{\infty}\right)$, we conclude that $\mathbb{E} \mathbb{R}(n)^{(*, *)}\left(R P^{\infty}\right) \simeq \mathbb{E} \mathbb{R}(n)^{(*, *)}[[u]] /[2](u)$.

At this point, let us recall the invertible class $y(n) \in \mathbb{E} \mathbb{R}(n)^{(-\lambda(n),-1)}[$ KW07a, Claim 4.1], where $\lambda(n)=2^{2 n+1}-2^{n+2}+1$. We have the $v_{k}^{\mathbb{E R}(n)} \in \mathbb{E} \mathbb{R}(n)^{\left(-2^{k}+1,-2^{k}+1\right)}$

and get elements $y(n)^{-2^{k}+1} v_{k}^{\mathbb{E R}(n)}=v_{k}^{E R(n)} \in E R^{\left(2^{k}-1\right)(\lambda(n)-1)}\left(S^{0}\right)$. We may normalize $u$ to be in degree $(1-\lambda(n), 0)$ by redefining $u$ as $u y(n)$. Using the first proposition, we get: 
Theorem 3.2. Let $\lambda(n)=2^{2 n+1}-2^{n+2}+1$. Then $E R(n)^{*}\left(R P^{\infty}\right)$ is isomorphic to $E R(n)^{*}[[u]] /([2](u))$ where $u \in E R(n)^{1-\lambda(n)}\left(R P^{\infty}\right)$ and the $v_{k}$ are replaced by $v_{k}^{E R(n)}$ $\in \operatorname{ER}(n)^{(\lambda(n)-1)\left(2^{k}-1\right)}\left(S^{0}\right)$.

We may also calculate the $E R(n)$ cohomology of spaces of the form $X \wedge R P^{\infty}$ using similar ideas. Let $X$ be a space with a trivial $\mathbb{Z} / 2$-action. As before, we may show that $\mathbb{E} \mathbb{R}(n)^{(*, *)}\left(X \wedge C P^{\infty}\right) \simeq \mathbb{E} \mathbb{R}(n)^{(*, *)}(X)[[u]]$. Again, we consider the real bundle $0 \times \gamma^{\otimes 2}$ over $X \wedge C P^{\infty}$ with Euler class [2] $(u)$. We have the Gysin sequence for this bundle:

$$
\begin{gathered}
\longrightarrow \mathbb{E} \mathbb{R}(n)^{(a-1, b-1)}(X)[[u]] \stackrel{[2](u)}{\longrightarrow} \mathbb{E} \mathbb{R}(n)^{(a, b)}(X)[[u]] \longrightarrow \\
\mathbb{E} \mathbb{R}(n)^{(a, b)}\left(X \wedge R \tilde{P}^{\infty}\right) \longrightarrow \mathbb{E} \mathbb{R}(n)^{(a, b-1)}(X)[[u]] \longrightarrow \cdots
\end{gathered}
$$

Since we know that $\mathbb{E} \mathbb{R}(n)^{(a, b)}\left(X \wedge R \tilde{P}^{\infty}\right)$ is isomorphic to $\mathbb{E} \mathbb{R}(n)^{(a, b)}\left(X \wedge R P^{\infty}\right)$ from an earlier proposition, we would be done provided we knew that [2](u) was not a zero divisor in $\mathbb{E} \mathbb{R}(n)^{(*, *)}(X)[[u]]$. For this we require an algebraic lemma (let $\left.v_{0}=2\right)$ :

Lemma 3.3. Let $M$ be a $\mathbb{E} \mathbb{R}(n)^{(*, *)}$-module such that $M$ has no infinitely $I$-divisible elements, where I is the ideal $\left(v_{0}, v_{1}^{\mathbb{E R}(n)}, \ldots, v_{n-1}^{\mathbb{E R}(n)}\right)$; i.e.

$$
\bigcap_{k} I^{k} M=0 .
$$

Then $[2](u)$ is not a zero divisor in $M[[u]]$.

Proof. Filter $M$ by submodules $0=\cap M^{k} \cdots \subset M^{2} \subset M^{1} \subset M^{0}=M$, where $M^{k}=$ $I^{k} M$. Notice that $v_{i}^{\mathbb{E R}(n)} M^{k} \subset M^{k+1}$ for $i<n$. Now let $f(u) \in M[[u]]$ be a power series with the property $f(u)[2](u)=0$, then working in $M / M^{1}[[u]]$, this equality reduces to $v_{n}^{\mathbb{E R}(n)} f(u) u^{2^{n}}=0$, which implies that $f(u)$ belongs to $M^{1}[[u]]$. Continuing with $M^{1} / M^{2}[[u]]$ and so forth, we conclude that $f(u) \in \cap_{k} M^{k}[[u]]=0$.

It follows from the above lemma and the Gysin sequence that

Theorem 3.4. Let $X$ be a space with the property that $E R(n)^{*}(X)$ has no infinitely

$v_{i}^{E R(n)}$-divisible elements for $i<n,\left(\right.$ e.g. $X$ is finite or $\left.X=R P^{\infty}\right)$. Let $u$ be the class defined earlier; then we have an isomorphism:

$$
E R(n)^{*}\left(X \wedge R P^{\infty}\right) \simeq E R(n)^{*}(X)[[u]] /([2](u))
$$

The proof, of course, is insignificantly different from Landweber's proof for $M U$ in $[\operatorname{Lan} 70]$.

\section{The Bockstein spectral sequence}

We begin with the stable cofibration (1.4) of [KW07a],

$$
\Sigma^{\lambda(n)} \operatorname{ER}(n) \stackrel{x}{\longrightarrow} E R(n) \longrightarrow E(n),
$$

where $x \in E R(n)^{-\lambda(n)}$ and $\lambda(n)=2^{2 n+1}-2^{n+2}+1$. 
The fibration gives us the long exact sequence (1.5). Our long exact sequence is an exact couple and so gives rise to a spectral sequence whose differentials give us the $x$-torsion. We have that $x^{2^{n+1}-1}=0,[\mathbf{H K 0 1}]$, so there are a finite number of differentials.

Most of the details of the spectral sequence are fairly straightforward but since we will make extensive use of it we want to be careful about its basics, so we collect them in a theorem. We will need complex conjugation. Since $E(n)$ is a complex orientable theory, it has a complex conjugation map on it that we denote by $c$. We always use reduced cohomology. We know that $2 x=0$, a simple fact that isn't necessary in the spectral sequence but should be kept in mind.

Theorem 4.2 (The Bockstein Spectral Sequence for $E R(n)^{*}(X)$ ).

(i) The exact couple of (1.5) gives a spectral sequence, $E^{r}$, of $E R(n)^{*}$-modules, starting with

$$
\begin{gathered}
E^{1} \simeq E(n)^{*}(X) . \\
E^{2^{n+1}}=0 .
\end{gathered}
$$

(iii) The targets of the differentials, $d^{r}$, represent the $x^{r}$-torsion generators of $E R(n)^{*}(X)$ as described below.

(iv) The degree of $d^{r}$ is $r \lambda(n)+1$.

(v) Filter $\operatorname{ER}(n)^{*}(X)$ by $K_{i}$, the kernel of $x^{i}$. Then

$$
\{0\}=K_{0} \subset K_{1} \subset K_{2} \subset \cdots \subset K_{2^{n+1}-1}=E R(n)^{*}(X) .
$$

(vi) Filter $M=E R(n)^{*}(X) / x E R(n)^{*}(X)$ by $M_{i}$ the image of $K_{i}$ so

$$
\begin{gathered}
\{0\}=M_{0} \subset M_{1} \subset M_{2} \subset \cdots \subset M_{2^{n+1}-1}=M . \\
M / M_{r-1} \longrightarrow E^{r}, \quad r \geqslant 1 \text { injects and } M_{r} / M_{r-1} \simeq \text { image } d^{r} .
\end{gathered}
$$

$$
\begin{gathered}
d^{r}(a b)=d^{r}(a) b+c(a) d^{r}(b) . \\
d^{1}(z)=v_{n}^{-\left(2^{n}-1\right)}(1-c)(z) \text { where } c\left(v_{i}\right)=-v_{i} . \\
\text { If } c(z)=z \in E^{1}, \text { then } d^{1}(z)=0 . \\
\text { If } c(z)=z \in E^{r}, \text { then } d^{r}\left(z^{2}\right)=0 .
\end{gathered}
$$

(x) The following are all vector spaces over $\mathbb{Z} /(2)$ :

$$
K_{j} / K_{i}, \quad M_{j} / M_{i}, \quad j \geqslant i>0, \text { and } E^{r}, \quad r>1 .
$$

Most of the theorem follows immediately from the basic properties of an exact couple and the fact that $x^{2^{n+1}-1}=0$. We defer those proofs we need until Section 6 after we have worked some simple examples.

Remark 4.3. Note that the image of $E R(n)^{*}(X) \rightarrow E(n)^{*}(X)$ gives the set of elements that are targets of differentials and therefore always have all the differentials trivial on them. Note also that anything in the image is invariant under the action of $c$. 
Remark 4.4. Since $\operatorname{ER}(n)^{*}(-)$ is $2^{n+2}\left(2^{n}-1\right)$-periodic, we will consider it as graded over $\mathbb{Z} /\left(2^{n+2}\left(2^{n}-1\right)\right)$. We have to do the same then with $E(n)^{*}(-)$ and we can accomplish this by setting the unit $v_{n}^{2^{n+1}}=1$ in the homotopy of $E(n)$.

Remark 4.5. Recall that $K O_{(2)}=E R(1)$. For a very simple warmup exercise we can compute the coefficient ring, $E R(1)^{*}\left(S^{0}\right)$, using the spectral sequence. Our $E^{1}$-term is $E(1)^{*}\left(S^{0}\right)$ made 8-periodic where $E(1)=K U_{(0)}$ so $E^{1}$ is just $\mathbb{Z}_{(2)}$ on generators $v_{1}^{i}, 0 \leqslant i<4$. We have that $c\left(v_{1}\right)=-v_{1}$ so

$$
d^{1}\left(v_{1}\right)=v_{1}^{-1}(1-c) v_{1}=v_{1}^{-1}\left(v_{1}+v_{1}\right)=2 .
$$

Similarly, $d^{1}\left(v_{1}^{3}\right)=2 v_{1}^{2}$. Since $c\left(v_{1}^{2}\right)=v_{1}^{2}$, we have $d^{1}\left(v_{1}^{2}\right)=0$. We have the $\mathbb{Z}_{(2)}$-free submodule generated by 2 and $2 v_{1}^{2}$ giving us our $x^{1}$-torsion. Give the element of $E R(1)^{*}\left(S^{0}\right)$ that maps to $2 v_{1}^{2}$ the name $\beta$. All that is left for our $E^{2}$-term is the $\mathbb{Z} /(2)$ vector space generated by 1 and $v_{1}^{2}$. They have degree 0 and 4 respectively. The only differentials we have left are $d^{2}$, which is odd degree so we don't have it, and $d^{3}$ which has degree 4 . Since we know 1 is in the image from $E R(1)^{*}\left(S^{0}\right)$, the differential must be $d^{3}\left(v_{1}^{2}\right)=1$. We have recovered the well known homotopy of $K O_{(0)}$. Our $x$ is really $\eta$ and we have $\eta^{3}=0$ on 1 . We have $2 \eta=0=\eta \beta$ and we have $\beta^{2}=4$. All of this read off from our spectral sequence for $\eta$ torsion.

\section{The spectral sequence for $\operatorname{ER}(2)^{*}(-)$}

In $[\mathbf{K W 0 7 b}]$ we describe the homotopy of $E R(2)$ in more detail than we need here. Our coefficient ring, $E R(2)^{*}\left(S^{0}\right)$, graded over $\mathbb{Z} /(48)$, is generated by elements, $x, w$, $\alpha, \alpha_{1}, \alpha_{2}$, and $\alpha_{3}$ of degrees $-17,-8,-32,-12,-24$, and -36 respectively. Some relations are given by $0=2 x=x^{7}=x^{3} w=x^{3} \alpha=x \alpha_{i}$ and $w^{2}=\alpha^{2}$. As a module over $\mathbb{Z}_{(2)}[\alpha]$, the homotopy can be described as having generators

$$
1, \quad w, \quad \alpha_{1}, \quad \alpha_{3} \text {, and } \alpha_{2}
$$

with one relation,

$$
\alpha \alpha_{2}=2 w
$$

copies of $\mathbb{Z} /(2)[\alpha]$ on generators

$$
x, \quad x^{2}, \quad x w, \quad x^{2} w
$$

and copies of $\mathbb{Z} /(2)$ on

$$
x^{3}, \quad x^{4}, \quad x^{5}, \quad x^{6} .
$$

The focus of our important computations which will rely on the spectral sequence will be for the theory $E R(2)^{*}(-)$. The homotopy of $E R(2)$ is non-trivial and taking a look at it from the perspective of the spectral sequence is well worth the effort, plus it helps us compute differentials in the future.

Our spectral sequence begins with $E^{1}=E(2)^{*}\left(S^{0}\right)$, which is just a free $\mathbb{Z}_{(2)}\left[v_{1}\right]$ module on a basis given by $v_{2}^{i}$ for $0 \leqslant i<8$. We know our $d^{1}$. We get:

$$
d^{1}\left(v_{2}^{2 s+1}\right)=v_{2}^{-3}(1-c)\left(v_{2}^{2 s+1}\right)=v_{2}^{-3} 2 v_{2}^{2 s+1}=2 v_{2}^{2 s-2} .
$$

Similarly, $d^{1}\left(v_{2}^{2 s}\right)=0$. This seems like a good start but if we multiply $v_{2}^{2 s+1}$ by $v_{1}$, the differential suddenly becomes zero because $c\left(v_{1} v_{2}^{2 s+1}\right)=v_{1} v_{2}^{2 s+1}$. Complicating 
matters even more, if we were to multiply by $v_{1}^{2}$ we would be back to our multiplication by $2 v_{2}^{-3}$. This is a problem that would persist in all of our computations for $E R(2)^{*}(X)$. We need a better way to deal with this.

Our solution to this problem is to introduce the element $\alpha$ discussed for (1.3). The image of $\alpha$ is $v_{1} v_{2}^{5} \in E(2)^{-32}\left(S^{0}\right)$ from $[\mathbf{K W 0 7 a}$ and [KW07b]. (We'll discuss $\alpha$ more in the next section.) Because $v_{2}$ is a unit, this is a good substitute for our plain $v_{1}$. Furthermore, it is invariant under $c$ because it is in the image of the map from $\operatorname{ER}(2)^{*}\left(S^{0}\right)$, or, we could just compute that $v_{1} v_{2}^{5}$ is invariant because it has an even number of $v$ 's. We now rewrite the homotopy of $E(2)$ as $\mathbb{Z}_{(2)}\left[\alpha, v_{2}^{ \pm 1}\right]$ but again set $v_{2}^{8}=1$.

We can go back to our computation of $d^{1}$ on $v_{2}^{2 s+1}$ where $E^{1}$ is now a free $\mathbb{Z}_{(2)}[\alpha]$ module on $v_{2}^{i}, 0 \leqslant i<8$. Now we could compute

$$
d^{1}\left(\alpha^{k} v_{2}^{2 s+1}\right)=d^{1}\left(\alpha^{k}\right) v_{2}^{2 s+1}+c\left(\alpha^{k}\right) d^{1}\left(v_{2}^{2 s+1}\right)=0+\alpha^{k} 2 v_{2}^{2 s-2},
$$

but this really follows automatically from the fact that the spectral sequence is a spectral sequence of $E R(2)^{*}$-modules.

After the $d^{1}$ in our spectral sequence for $E R(2)^{*}\left(S^{0}\right)$, all we have left for $E^{2}$ is the free $\mathbb{Z} /(2)[\alpha]$-module with basis given by $\left\{1, v_{2}^{2}, v_{2}^{4}, v_{2}^{6}\right\}$. We give names to the elements of $\operatorname{ER}(2)^{*}\left(S^{0}\right)$ that must be $x^{1}$-torsion and map to $2 v_{2}^{2 s}$. Let $\alpha_{i} \in E R(2)^{-12 i}\left(S^{0}\right)$ map to $2 v_{2}^{2 i}$ where $\alpha_{0}=2$. In $E R(2)^{*}\left(S^{0}\right)$, these elements generate a free $x^{1}$-torsion submodule over $\mathbb{Z}_{(2)}[\alpha]$.

Since all our remaining elements in $E^{2}$ are in even degrees, we can only have odd differentials since the even ones have odd degrees. Our choices are $d^{3}, d^{5}$, and $d^{7}$. The degree of $d^{5}$ is 38 . If we look at our $E^{2}$ in degrees modulo 16 , we find that we only have elements in degrees $0,4,8$, and 12 . The mod 16 degree of $d^{5}$ is 6 and so must be zero. Note also that we must have two non-trivial differentials because $v_{2}^{4}=\left(v_{2}^{2}\right)^{2}$ and we can apply our Theorem 4.2 to show that our first new differential must be trivial on this.

We need the differentials on the coefficients because we will use them regularly in our other computations. We also want to demonstrate how much information can be extracted from the spectral sequence without much input. In our present case all we have done is replace $v_{1}$ with the invariant $\alpha=v_{1} v_{2}^{5}$. Proceeding, we must have a $d^{3}$ and it must be non-trivial on $v_{2}^{2}$ and $v_{2}^{6}$. The degree of $d^{3}$ is 4 , (remember, we are graded over $\mathbb{Z} /(48))$, so $d^{3}\left(v_{2}^{2}\right)=\alpha^{3 k+1} v_{2}^{4}$ for some $k$ for degree reasons. Multiply by $v_{2}^{4}$ to get $d^{3}\left(v_{2}^{6}\right)=\alpha^{3 k+1} 1$. Unfortunately, we don't know $k$. However, if we keep going, we can compute our $E^{4}=E^{7}$. It is just $\alpha^{i} 1$ and $\alpha^{i} v_{2}^{4}$ for $0 \leqslant i \leqslant 3 k$. Since we know that $\alpha^{i} 1$ is in the image, it must be the target of differentials and all that is left is $d^{7}\left(\alpha^{i} v_{2}^{4}\right)=\alpha^{i} 1$. Since $E^{8}$ must be zero, this is forced.

At this stage we have to introduce a fact, namely that $x^{3} \alpha=0[\mathbf{K W 0 7} \mathbf{b}]$. That forces our $k$ above to be zero and our $d^{7}$ to just be $d^{7}\left(v_{2}^{4}\right)=1$. Name the element that maps to $\alpha v_{2}^{4}, w \in E R(2)^{-8}\left(S^{0}\right)$. Our $x^{3}$-torsion elements are given by $\alpha^{k}$ and $w \alpha^{k}$. Finally, our only $x^{7}$-torsion element is 1 .

In order to do this computation the only thing we had to use that didn't come directly from the spectral sequence was the fact that $x^{3} \alpha=0$. We can recover most, if not all, of the ring structure by looking at the image of $E R(2)^{*}\left(S^{0}\right)$ in $E(2)^{*}\left(S^{0}\right)$ (for example, $\alpha_{2}^{2}=4$ and $\alpha_{2} \alpha=2 w$ ). 
We want another relation. Note that in our spectral sequence we have

$$
w^{2}=\left(\alpha v_{2}^{4}\right)^{2}=\alpha^{2} v_{2}^{8}=\alpha^{2} .
$$

This is only modulo $x$ but this is in degree -16 . The degree of $x$ is -17 and there are no elements at all in degree 1 , so there is no $z$ such that we could possibly have $x z+w^{2}=\alpha^{2}$, so this relation, $w^{2}=\alpha^{2}$ must be true on the nose.

From our theorem and our computation:

Proposition 5.1. In the Bockstein spectral sequence for $E R(2)^{*}(X)$, the map $d^{1}$ is an $E R(2)^{*} /(x)$-module map. The differentials $d^{2}$ and $d^{3}$ are $\mathbb{Z} /(2)[\alpha, w] /\left(w^{2}=\alpha^{2}\right)$ module maps but $d^{4}, d^{5}, d^{6}$, and $d^{7}$ are only $\mathbb{Z} /(2)$-module maps.

Proof. Of course all of these differentials are really still $E R(2)^{*}$-module maps but some of the elements of $E R(2)^{*}$ are zero in $E^{r}$. For example, the $\alpha_{i}$ and 2 are all zero in $E^{2}$. All that is left then is $\mathbb{Z} /(2)[\alpha, w] /\left(w^{2}=\alpha^{2}\right)$ but $w$ and $\alpha$ go to zero in $E^{4}$ leaving only $\mathbb{Z} /(2)$.

Our Bockstein spectral sequences will be modules over $E R(2)^{*}$. We collect some of the facts we will use repeatedly:

\section{Proposition 5.2.}

$$
\begin{gathered}
d^{1}\left(v_{2}^{2 s+1}\right)=2 v_{2}^{2 s-2} ; \quad d^{3}\left(v_{2}^{4 s-2}\right)=\alpha v_{2}^{4 s} ; \quad d^{7}\left(v_{2}^{4}\right)=1 ; \\
d^{1}\left(v_{2}^{2 s}\right)=0 ; \quad d^{2}\left(v_{2}^{2 s}\right)=0 \\
d^{r}\left(v_{2}^{4 s}\right)=0 ; \quad 3 \leqslant r<7 .
\end{gathered}
$$

\section{Proof of Theorem 4.2}

The spectral sequence obtained from (1.5) is a classic example of an exact couple. Everything but the facts about the differentials is automatic. Even the product rule for $d^{r}$ follows if we know it for $d^{1}$. It is as if Bill Massey consulted us about what we needed before he wrote [Mas54]. We have complex conjugation for our involution on $E(n)^{*}(X)$ and the trivial involution, i.e. the identity, on $E R(n)^{*}(X)$. Our situation then fits [Mas54] exactly. Assuming our formula for $d^{1}$ we confirm the product formula for it:

$$
\begin{aligned}
d^{1}(a b) & =v_{n}^{-\left(2^{n}-1\right)}(1-c)(a b)=v_{n}^{-\left(2^{n}-1\right)}(a b-c(a b))=v_{n}^{-\left(2^{n}-1\right)}(a b-c(a) c(b)) \\
& =v_{n}^{-\left(2^{n}-1\right)}((a-c(a)) b+c(a)(b-c(b)))=d^{1}(a) b+c(a) d^{1}(b) .
\end{aligned}
$$

We prove the part that assumes $c(z)=z$ :

$$
d^{1}(z)=v_{n}^{-\left(2^{n}-1\right)}(1-c)(z)=v_{n}^{-\left(2^{n}-1\right)}(z-z)=0 .
$$

For the second case, $d^{1}\left(z^{2}\right)=0$ because $z^{2}$ is invariant under $c$. For $r>1$,

$$
d^{r}\left(z^{2}\right)=d^{r}(z) z+c(z) d^{r}(z)=d^{r}(z) z+z d^{r}(z)=2 z d^{r}(z),
$$

which is zero since we are working modulo 2 for $r>1$.

All that remains is to get our formula for $d^{1}$ and prove our statements about mod 2 vector spaces. Let's continue to assume our formula for $d^{1}$ and show our $\mathbb{Z} /(2)$ 
vector spaces. First, we note that $2 x=0$ so $2 E R(n)^{*}(X) \subset K_{1}$. This is all we need to show that

$$
K_{j} / K_{i}, \text { and } M_{j} / M_{i}, \quad j \geqslant i>0
$$

are $\mathbb{Z} /(2)$ vector spaces. To show that $E^{r}, r>1$, is a $\mathbb{Z} /(2)$ vector space, it is enough to show it for $E^{2}$. We start with an arbitrary element $y \in E^{1}$ with $2 y \neq 0$. Obviously, if $d^{1}(y) \neq 0$ this situation does not persist to $E^{2}$ so we can assume that $d^{1}(y)=0$. First we need

$$
d^{1}\left(v_{n}^{2^{n}-1}\right)=v_{n}^{-\left(2^{n}-1\right)}(1-c) v_{n}^{2^{n}-1}=v_{n}^{-\left(2^{n}-1\right)}\left(v_{n}^{2^{n}-1}+v_{n}^{2^{n}-1}\right)=2
$$

(which, by the way, shows $2 x=0$ ). Consider the element $v_{2}^{2^{n}-1} y$. We have:

$$
d^{1}\left(v_{2}^{2^{n}-1} y\right)=d^{1}\left(v_{2}^{2^{n}-1}\right) y+c\left(v_{2}^{2^{n}-1}\right) d^{1}(y)=2 y+0 .
$$

Thus no multiplication by 2 survives to $E^{2}$, which concludes our proof.

We have only one thing left to do, and that is to prove the formula $d^{1}=$ $v_{n}^{-\left(2^{n}-1\right)}(1-c)$. We've put this off till last because it requires a review of the source of our fibration. This also gives us a chance to describe some of the general properties of $E R(n)^{*}(X)$. In $[\mathbf{K W 0 7 a}]$ we have bigraded spaces, $\mathbb{E}(n)_{a, b}$ with $b=0$ giving our standard $\Omega$ spectrum for $E(n)$. Likewise we have $\mathbb{E} \mathbb{R}(n)_{a, b}$ with $b=0$ giving our $\Omega$ spectrum for $E R(n)$.

There is ample opportunity for confusion here. Before we proceed, let's do a little review of all the elements named $v_{k}$. Our unadorned element is

$$
v_{k} \in E(n)^{-2\left(2^{k}-1\right)}\left(S^{0}\right)=\mathbb{E}(n)^{-2\left(2^{k}-1\right), 0}\left(S^{0}\right),
$$

where $\mathbb{E}(n)$ is the bigraded equivariant spectrum with complex conjugation, $c$, acting on it. The element

$$
v_{k}^{\mathbb{E}(n)} \in \mathbb{E}(n)^{-\left(2^{k}-1\right),-\left(2^{k}-1\right)}\left(S^{0}\right)
$$

is invariant under the action of $c$ and gives rise to

$$
v_{k}^{\mathbb{E R}(n)} \in \mathbb{E} \mathbb{R}(n)^{-\left(2^{k}-1\right),-\left(2^{k}-1\right)}\left(S^{0}\right) .
$$

We have an element

$$
\sigma \in \pi_{0}\left(\mathbb{E}(n)_{1,-1}\right)
$$

with a non-trivial $\mathbb{Z} /(2)$ action on it. However, the element $\sigma^{2^{n+1}}$ lifts to a unit in $\pi_{0}\left(\mathbb{E} \mathbb{R}(n)_{2^{n+1},-2^{n+1}}\right)=\mathbb{E} \mathbb{R}(n)^{2^{n+1},-2^{n+1}}\left(S^{0}\right)$.

The first thing we want to show is how the invariant $v_{k}^{\mathbb{E}(n)} \in \mathbb{E}(n)^{-2^{k}+1,-2^{k}+1}\left(S^{0}\right)$ is connected to our $v_{k} \in \mathbb{E}(n)^{-2\left(2^{k}-1\right), 0}\left(S^{0}\right) \equiv E(n)^{-2\left(2^{k}-1\right)}\left(S^{0}\right)$. We have

$$
v_{k}=v_{k}^{\mathbb{E}(n)} \sigma^{-2^{k}+1} \text {. }
$$

Since there are an odd number of $\sigma$ 's, we get our $c\left(v_{k}\right)=-v_{k}$.

In [KW07a, Claim 4.1] we produced an invertible homotopy element

$$
y(n)=\left(v_{n}^{\mathbb{E R}(n)}\right)^{2^{n}-1} \sigma^{-2^{n+1}\left(2^{n-1}-1\right)} \in \mathbb{E} \mathbb{R}(n)^{-\lambda(n),-1}\left(S^{0}\right),
$$

and multiplication by it gives an isomorphism

$$
\mathbb{E} \mathbb{R}(n)^{a+1+\lambda(n), b}(X) \simeq \mathbb{E} \mathbb{R}(n)^{a+1, b-1}(X) .
$$


Note that

$$
y(n)^{-1}=\left(v_{n}^{\mathbb{E R}(n)}\right)^{-\left(2^{n}-1\right)} \sigma^{2^{n+1}\left(2^{n-1}-1\right)}=\left(v_{n}^{\mathbb{E} \mathbb{R}(n)} \sigma^{-\left(2^{n}-1\right)}\right)^{-\left(2^{n}-1\right)} \sigma^{-1},
$$

which reduces to $v_{n}^{-\left(2^{n}-1\right)} \sigma^{-1}$ in $\mathbb{E}(n)^{\lambda(n),+1}\left(S^{0}\right)$.

We get a new element

$$
v_{k}^{E R(n)}=v_{k}^{\mathbb{E R}(n)} y(n)^{-2^{k}+1} \in E R(n)^{(\lambda(n)-1)\left(2^{k}-1\right)}\left(S^{0}\right),
$$

which reduces, in $E(n)^{(\lambda(n)-1)\left(2^{k}-1\right)}\left(S^{0}\right)$, to $v_{k} v_{n}^{-\left(2^{n}-1\right)\left(2^{k}-1\right)}$.

In order for the map of $E R(n)$ to $E(n)$ to work nicely we would replace the element $v_{k} \in E(n)^{*}\left(S^{0}\right)$ with $v_{k} v_{n}^{-\left(2^{n}-1\right)\left(2^{k}-1\right)}$. In particular, when $k=1$ and $n=2$, we did this in the last section when we renamed this element $\alpha$.

The element $v_{n}^{\mathbb{E R}(n)}$ is a unit and so is $\sigma^{2^{n+1}}$. Consequently, so is

$$
\left(v_{n}^{\mathbb{E R}(n)}\right)^{2^{n+1}} \sigma^{-2^{n+1}\left(2^{n}-1\right)}=\left(v_{n}^{\mathbb{E R}(n)} \sigma^{-\left(2^{n}-1\right)}\right)^{2^{n+1}},
$$

and this is the periodicity element for $E R(n)^{*}(-) \equiv \mathbb{E} \mathbb{R}(n)^{*, 0}(-)$ and it maps to $v_{n}^{2^{n+1}}$ in $E(n)^{*}\left(S^{0}\right)$.

In $[\mathbf{K W 0 7 a}]$ the fibration actually proven is

$$
\mathbb{E} \mathbb{R}(n)_{a, b-1} \longrightarrow \mathbb{E} \mathbb{R}(n)_{a, b} \longrightarrow \mathbb{E}(n)_{a, b}
$$

The map, $\partial$,

$$
\mathbb{E}(n)_{a, b} \longrightarrow \mathbb{E} \mathbb{R}(n)_{a+1, b-1} \longrightarrow \mathbb{E}(n)_{a+1, b-1}
$$

is evaluated in [KW07a, Proposition 1.6] as $1-c$ with the understanding that the two ends are homeomorphic because they are both just loops on $\mathbb{E}(n)_{a+1, b}$ with different $\mathbb{Z} /(2)$ actions. Multiplication by our $\sigma$ gives this homeomorphism. So, implicit in [KW07a, Proposition 1.6] is

$$
\sigma^{-1} \partial=1-c
$$

This map corresponds somewhat to our first differential. However, we work with the spectra $E R(n)$ and $E(n)$. Our boundary map, i.e. $d^{1}$, is

$$
E(n)_{a} \longrightarrow E R(n)_{a+\lambda(n)+1} \longrightarrow E(n)_{a+\lambda(n)+1} \text {. }
$$

To finish off our $d^{1}$ we need the diagram:

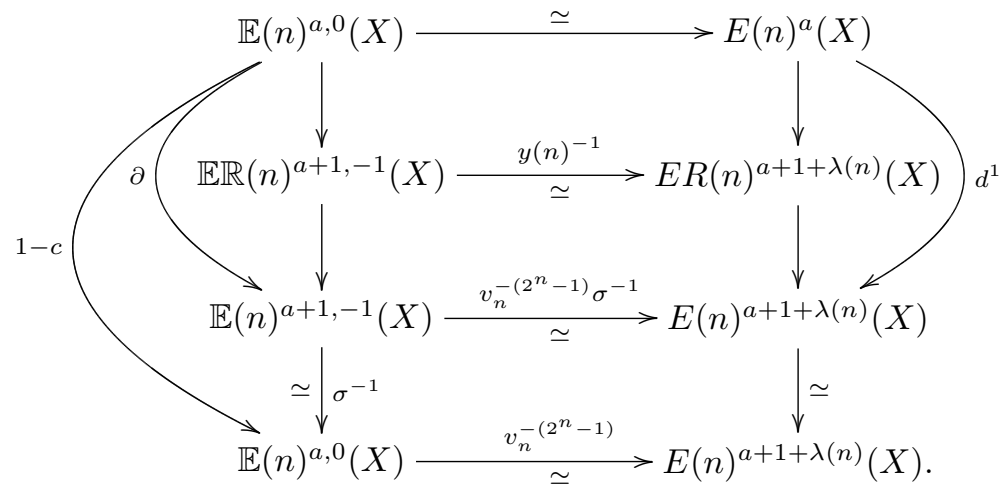




$$
\begin{aligned}
& \sigma^{-1} \partial=(1-c), \text { so } \\
& \qquad d^{1}=\left(v_{n}^{-\left(2^{n}-1\right)} \sigma^{-1}\right) \partial=v_{n}^{-\left(2^{n}-1\right)}\left(\sigma^{-1} \partial\right)=v_{n}^{-\left(2^{n}-1\right)}(1-c) .
\end{aligned}
$$

This concludes our proof.

\section{Notational conventions}

Our descriptions of groups are usually by giving a "2-adic basis;" i.e. a set of elements such that any element in our group is written as a unique finite sum of these elements with coefficients 0 or 1 . For example, if we have $\mathbb{Z} /\left(2^{n}\right)$ generated by $u$ with the relation $2 u=u^{2}$, our 2 -adic basis would be $u^{j}, 0<j \leqslant n$. In the case of infinite dimensional spaces we can have infinite sums but care must be taken about the topology.

We frequently write our list of elements as efficiently as possible by using notation such as $x^{\{1,2\}}$ and $x^{\{0-2\}}$ to indicate the obvious list of elements; $x$ and $x^{2}$ in the first case and 1, x, and $x^{2}$ in the second case. This notation will be used in both superscripts and subscripts.

Whenever we use $\epsilon$, we mean it can be either 0 or 1 .

Whenever we give names to new elements, the subscript given as part of the name is also the degree of the element.

\section{The Bockstein spectral sequence for $\operatorname{ER}(2)^{*}\left(R P^{\infty}\right)$}

We begin by computing $E R(2)^{*}\left(R P^{\infty}\right)$ using the Bockstein spectral sequence. In principle, we already know this from Theorem 3.2. Note that until we start our work with products, many of our Bockstein spectral sequences are of even degree. Our even differentials, $d^{2 r}$, are odd degree so they are all zero. This leaves us with only $d^{\{1,3,5,7\}}$.

We remind the reader that the image of our $u \in E R(2)^{-16}\left(R P^{\infty}\right)$ is an element we also call $u \in E(2)^{-16}\left(R P^{\infty}\right)$, which is really $v_{2}^{3} x_{2}$ where $x_{2}$ is our usual 2-dimensional generator and $v_{2}$ is invertible of degree -6 . Likewise we replace the usual $v_{1} \in$ $E(2)^{-2}$ with $v_{2}^{5} v_{1}=\alpha \in E(2)^{-32}$ which comes from $\alpha \in E R(2)^{-32}$. The element $w \in$ $E R(2)^{-8}$ maps to $\alpha v_{2}^{4}=v_{2} v_{1} \in E(2)^{-8}$. These changes are necessary because $x_{2}$ and $v_{1}$ are not in the image but elements that differ only by a unit are.

Theorem 8.1 (The Bockstein spectral sequence for $E R(2)^{*}\left(R P^{\infty}\right)$ ).

$E^{1}=E(2)^{*}\left(R P^{\infty}\right)$ has 2-adic basis

$$
\begin{gathered}
v_{2}^{i} \alpha^{k} u^{j}, \quad 0 \leqslant i<8, \quad 0 \leqslant k, \quad 1 \leqslant j \\
d^{1}\left(v_{2}^{2 s-5} \alpha^{k} u^{j}\right)=2 v_{2}^{2 s} \alpha^{k} u^{j} \equiv v_{2}^{2 s} \alpha^{k+1} u^{j+1}
\end{gathered}
$$

modulo higher powers of $u$.

$E^{2}=E^{3}$ is given by:

$$
\begin{gathered}
v_{2}^{2 s} \alpha^{k} u ; \quad v_{2}^{2 s} u^{j}, \quad 2 \leqslant j, \quad 0 \leqslant s<4, \quad 0 \leqslant k \\
d^{3}\left(v_{2}^{4 s-2} \alpha^{k} u\right)=v_{2}^{4 s} \alpha^{k+1} u
\end{gathered}
$$


and for $2 \leqslant j$,

$$
d^{3}\left(v_{2}^{4 s-2} u^{j}\right)=v_{2}^{4 s} \alpha u^{j} \equiv v_{2}^{4 s} u^{j+2}
$$

modulo higher powers of $u$.

$E^{4}=E^{5}=E^{6}=E^{7}$ is given by

$$
\begin{array}{r}
v_{2}^{4} u^{\{1-3\}} \text { and } u^{\{1-3\}} \\
d^{7}\left(v_{2}^{4} u^{\{1-3\}}\right)=u^{\{1-3\}} .
\end{array}
$$

The $x^{1}$-torsion generators are given by:

$$
\alpha_{i} \alpha^{k} u^{j}, \quad 0 \leqslant i, \quad 0 \leqslant k, \quad 1 \leqslant j
$$

where $\alpha_{0}=2$.

The $x^{3}$-torsion generators are given by:

$$
w^{\epsilon} \alpha^{k} u, \quad \epsilon+k>0 ; \quad w u^{j}, \quad 1<j ; \text { and } u^{j}, \quad 3<j .
$$

The only $x^{7}$-torsion generators are

$$
u^{\{1-3\}} \text {. }
$$

Remark 8.2. This is consistent with the description in Theorem 3.2. Because $2 x=0$, $x$ times the relation $0=2 u+{ }_{F} \alpha u^{2}+{ }_{F} u^{4}$ gives us $0=x\left(\alpha u^{2}+{ }_{F} u^{4}\right)$. This explains why no $\alpha u^{2}$ shows up in our description. From the point of view of $x$-torsion it can be replaced with $u^{4}$ plus other terms. Likewise, if we multiply by $x^{3}$ and use the relation $x^{3} \alpha=0$ we end up with $x^{3} u^{4}=0$.

Proof. The proof is straightforward. Since $u \in E(2)^{*}\left(R P^{\infty}\right)$ is in the image from $E R(2)^{*}\left(R P^{\infty}\right)$, our differentials commute with multiplication by $u$ (from the product formula). They also commute with multiplication by $\alpha$. We also have, from our computation of the spectral sequence for $E R(2)^{*}\left(S^{0}\right)$ the differentials of Proposition 5.2. The $d^{1}$ differential creates a relation coming from our relation $0=2 u+{ }_{F} \alpha u^{2}{ }_{F} u^{4}$ when $2 u$ is set to zero. So, in $E^{2}$, we have $\alpha u^{2} \equiv u^{4}$ modulo higher powers of $u$. This explains some of our $d^{3}$. All of our differentials follow.

We use the map $E R(2)^{*}\left(S^{0}\right) \rightarrow E(2)^{*}\left(S^{0}\right)$ which takes $\alpha_{i} \rightarrow 2 v_{2}^{2 i}$ and $w \rightarrow v_{2}^{4} \alpha$ to identify the $x^{r}$-torsion generators.

Corollary 8.3. The map $E R(2)^{*}(-) \rightarrow E(2)^{*}(-)$ induces an isomorphism

$$
E R(2)^{16 *}\left(R P^{\infty}\right) \longrightarrow E(2)^{16 *}\left(R P^{\infty}\right) .
$$

Both have 2-adic bases given by $\alpha^{k} u^{j}$.

Proof. We have, for $E(2)^{16 *}\left(R P^{\infty}\right)$, a 2 -adic basis, $\alpha^{i} u^{j}$. Since $\alpha$ and $u$ both come from $E R(2)^{*}\left(R P^{\infty}\right)$, we have a surjection. From the Bockstein spectral sequence for $E R(2)^{*}\left(R P^{\infty}\right)$ we can just read off all of the elements in degree $16 *$. From the $x^{1}$ torsion we have $\alpha_{0} \alpha^{k} u^{j}$ where $\alpha_{0}=2$. These elements are, modulo higher filtration, $\alpha^{k+1} u^{j+1}$. From the $x^{3}$-torsion we have $\alpha^{k} u$ and $u^{j}$ for $k>0$ and $j>3$. There are elements in degree $8 \bmod 16$ but with the degree of $x$ equal to -17 they do not give rise to any more degree $16 *$ elements. Likewise for the $x^{7}$-torsion where we pick up only $u^{\{1-3\}}$. Altogether we have $\alpha^{i} u^{j}$, the same as for $E(2)^{16 *}\left(R P^{\infty}\right)$. 
Remark 8.4. In the next paper we will need the slightly more delicate fact that $E R(2)^{16 *+8}\left(R P^{\infty}\right)$ injects into $E(2)^{16 *+8}\left(R P^{\infty}\right)$.

\section{9. $\quad E R(2)^{*}\left(R P^{2}\right)$}

To start our computation of $E R(2)^{*}\left(R P^{2}\right)$ we revert to the Atiyah-Hirzebruch spectral sequence. Recall the homotopy of $E R(2)$ from the beginning of Section 5 . The Atiyah-Hirzebruch spectral sequence has elements only in filtrations 1 and 2 . In filtration 1 we have $w^{\epsilon} \alpha^{k} x^{\{1,2\}} x_{1}$ and $x^{\{3-6\}} x_{1}$. In filtration 2 we have $w^{\epsilon} \alpha^{k} x^{\{0,1,2\}} x_{2}$, $x^{\{3-6\}} x_{2}, \alpha_{\{1,3\}} \alpha^{k} x_{2}$ and $\alpha_{2} x_{2}$. Since all differentials increase filtration by at least 2 , the spectral sequence collapses. As $E R(2)^{*}$-modules this is generated by elements we call $z_{-16}$ represented by $x x_{1}$ (recall that the degree of $x$ is -17 ) and $z_{2}$ represented by $x_{2}$. Remember, of course, that we are working in degrees indexed by $\mathbb{Z} /(48)$ for $E R(2)^{*}(-)$ and $E(2)^{*}(-)$.

There is a surprising amount of detail to be had in $E R(2)^{*}\left(R P^{2}\right)$. We distill what we need down to:

Theorem 9.1. We have elements $z_{2}$ and $z_{-16}=u \in E R(2)^{*}\left(R P^{2}\right)$. A 2-adic basis for $E R(2)^{*}\left(R P^{2}\right)$ is given by $x^{\{0-2\}} w^{\epsilon} \alpha^{k} z_{-16}, x^{\{3-6\}} z_{-16}, x^{\{0-2\}} w^{\epsilon} \alpha^{k} z_{2}$, and $x^{\{3-6\}} z_{2}$ where $2 w^{\epsilon} \alpha^{k} z_{-16}=x^{2} w^{\epsilon} \alpha^{k+1} z_{2}, u^{2}=x^{2} z_{2}, u^{3}=0=u z_{2}, x^{6} z_{-16}=\alpha_{2} z_{2}$, $x^{2} \alpha^{k+1} z_{-16}=\alpha_{3} \alpha^{k} z_{2}$, and $x^{2} w \alpha^{k} z_{-16}=\alpha_{1} \alpha^{k} z_{2}$.

Proof. We consider the commuting diagram:

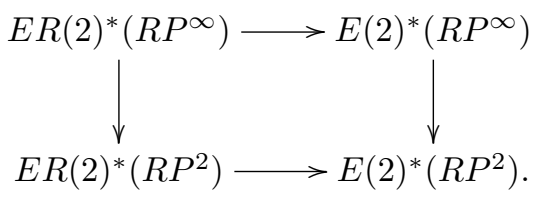

We know that the $u \in E R(2)^{-16}\left(R P^{\infty}\right)$ factors through $E(2)^{-16}\left(R P^{\infty}\right)$ to $u \in$ $E(2)^{-16}\left(R P^{2}\right)$ and so we must have $0 \neq u \in E R(2)^{-16}\left(R P^{2}\right)$ as well. The only element that could represent this $u$ is $x x_{1}=z_{-16}$. That means $u^{2}$ is represented by $\left(x x_{1}\right)^{2}=x^{2} x_{2}$. We have $u^{3}$ and $u z_{2}$ in filtration 3 , which is zero. Recalling our relation, we have $0=2 u+{ }_{F} \alpha u^{2}+{ }_{F} u^{4}$. This simplifies because $u^{4}$ would have to be in at least the 4 th filtration but everything above the 2nd filtration is zero. Thus our relation is $0=2 u+{ }_{F} \alpha u^{2}$, but since $2 u^{2}$ must be in filtration 3 or higher this is only $0=2 u+\alpha u^{2}$ and since filtration 2 is all modulo (2) we can just as well use $2 u=\alpha u^{2}$. From this, of course, we get $2 w^{\epsilon} \alpha^{k} u=w^{\epsilon} \alpha^{k+1} u^{2}$, or, really, $2 w^{\epsilon} \alpha^{k} z_{-16}=x^{2} w^{\epsilon} \alpha^{k+1} z_{2}$.

We still don't know all we want to yet. The $E R(2)$ cohomology of this simple Moore space is unnecessarily complex. We can solve the next level of problem by looking at the long exact sequence:

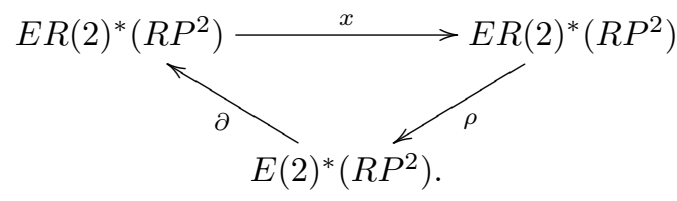


$E(2)^{*}\left(R P^{2}\right)$ is given by $v_{2}^{i} \alpha^{k} u$ and is a $\mathbb{Z} /(2)$ vector space. We know that $\alpha_{i} \rightarrow$ $2 v_{2}^{2 i}$ so $\alpha_{i} z_{2}$ must map to zero. That means these elements are divisible by $x$. The only candidates, mainly for degree reasons, are $x^{6} z_{-16}=\alpha_{2} z_{2}, x^{2} \alpha z_{-16}=\alpha_{3} z_{2}$ and $x^{2} w z_{-16}=\alpha_{1} z_{2}$.

The long exact sequence (9.2) gives the Bockstein spectral sequence, so as long as we are using it, we may as well do it using the Bockstein spectral sequence directly. We will be working with the Bockstein spectral sequence in general and we need to set this up for our future calculations.

$E^{1} \simeq E(2)^{*}\left(R P^{2}\right)$ for the Bockstein spectral sequence for $E R(2)^{*}\left(R P^{2}\right)$ where we are working with our usual 48-periodic $E(2)$. This $E^{1}$ is: $v_{2}^{i} \alpha^{k} u \quad 0 \leqslant i<8$ which is a vector space over $\mathbb{Z} /(2)$. We know, from the Atiyah-Hirzebruch spectral sequence, that we have two $E R(2)^{*}$ generators, $z_{-16}$ and $z_{2}$, that must map nontrivially to $E(2) *\left(R P^{2}\right)$.

We also know that $z_{-16} \rightarrow u$. Thus all differentials must be trivial on $u$. We use the product formula and the fact that $d^{1}$ times even powers of $v_{2}$ is zero and $d^{1}$ on odd powers of $v_{2}$ gives 2 times an even power which is also zero since we are working modulo 2. So

$$
d^{1}\left(v_{2}^{s} u\right)=d^{1}\left(v_{2}^{s}\right) u+c\left(v_{2}^{s}\right) d^{1}(u)=0+0
$$

$d^{1}$ is trivial in our spectral sequence. The differential $d^{2}$ is trivial because it is odd degree.

Again we can use the product rule and $d^{3}\left(v_{2}^{\{2,6\}}\right)=\alpha v_{2}^{\{4,0\}}$ to get

$$
d^{3}\left(v_{2}^{\{2,6\}} \alpha^{k} u\right)=\alpha^{k+1} v_{2}^{\{4,0\}} u .
$$

We need to worry about the elements $v_{2}^{2 s+1} u$.

For purely degree reasons the image of $z_{2}$ must go to a finite sum of $\alpha^{3 k} v_{2}^{5} u$ elements. All differentials must be trivial on this image element, in particular, $d^{3}$. Since there is no $\alpha$ torsion and $d^{3}$ commutes with $\alpha$, this implies that $d^{3}\left(v_{2}^{5} u\right)=0$.

As before,

$$
d^{3}\left(v_{2}^{\{2,6\}} \alpha^{k} v_{2}^{5} u\right)=v_{2}^{\{4,0\}} \alpha^{k+1} v_{2}^{5} u
$$

and the image of $z_{2}$ must be $v_{2}^{5} u$ (we may have to alter our choice of $z_{2}$ a bit for this) with $\alpha^{k} z_{2} \rightarrow v_{2}^{5} \alpha^{k} u$ and $w \alpha^{k} z_{2} \rightarrow v_{2} \alpha^{k+1} u$ (recall that $w \rightarrow v_{2}^{4} \alpha$ ).

Our $E^{4}$-term is quite small, just

$$
v_{2}^{\{0,1,4,5\}} u
$$

Because $v_{2}^{\{0,5\}} u$ are both in the image, degree reasons force us to have no $d^{4}, d^{5}$, or $d^{6}$, but we see that $d^{7}\left(v_{2}^{4} u\right)=u$ and $d^{7}\left(v_{2} u\right)=v_{2}^{5} u$.

From the Bockstein spectral sequence perspective we have no $x^{1}$-torsion generators. Our $x^{3}$-torsion generators are given by $w^{\epsilon} \alpha^{k} z_{\{2,-16\}}$ with $\epsilon+k>0$ and, finally, our $x^{7}$-torsion generators are $z_{2}$ and $z_{-16}$, or, as we write for efficiency's sake, $z_{\{2,-16\}}$. 
We can solve our Atiyah-Hirzebruch spectral sequence extension problems yet again using this approach. We now know we must have $x^{6} z_{-16} \neq 0$. The only possibility is for $x^{6} z_{-16}=\alpha_{2} z_{2}$. Likewise, we know that $x^{2}$ must be nonzero on all the $w^{\epsilon} \alpha^{k} z_{-16}$ when $\epsilon+k>0$. Since they only have $x$ times them nonzero in filtration 1 of the Atiyah-Hirzebruch spectral sequence, these elements must all be in filtration 2 and we get the answer we have already obtained from the long exact sequence.

The Moore space will be our basic building block.

Corollary 9.3. Consider the cofibration:

$$
S^{1} \longrightarrow R P^{2} \longrightarrow S^{2}
$$

The long exact sequence

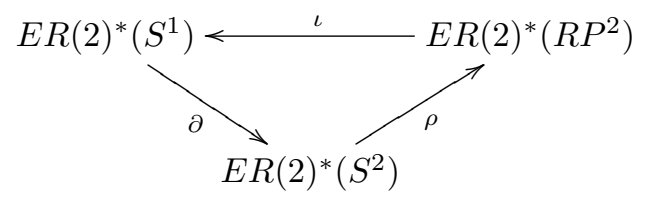

is given by $\partial\left(\iota_{1}\right)=2 \iota_{2}, \rho\left(\iota_{2}\right)=z_{2}$, and $\iota^{*}(u)=x \iota_{1}$.

\section{The spectral sequence for $\operatorname{ER}(2)^{*}\left(R P^{2 n} / R P^{2 n-2}\right)$}

For our computation we need the Bockstein spectral sequence in detail. Stating the complete Bockstein spectral sequence for even a simple space is highly technical. We need to give the $E^{r}$-terms for $r$ from 1 to 7 , compute the differentials and find corresponding $x^{r}$-torsion generators in $E R(2)^{*}(X)$ that map to the image of $d^{r}$. After this is done, we have to solve extension problems and locate any special elements of interest to us. Normally we won't need to do all of this, but as these spaces are our basic building blocks we need to know them quite well.

We have already done the case of $E R(2)^{*}\left(R P^{2}\right)$ and we know that

$$
\Sigma^{2 n-2} E R(2)^{*}\left(R P^{2}\right) \simeq E R(2)^{*}\left(R P^{2 n} / R P^{2 n-2}\right),
$$

so we can just write down the answer. Note, in particular, that the right hand side inherits a multiplication by $u$ from the left hand side.

Theorem 10.1. We have elements $z_{2 n-18}$ and $z_{2 n} \in E R(2)^{*}\left(R P^{2 n} / R P^{2 n-2}\right)$. $A$ 2-adic basis for $\operatorname{ER}(2)^{*}\left(R P^{2 n} / R P^{2 n-2}\right)$ is given by elements $x^{\{0-2\}} w^{\epsilon} \alpha^{k} z_{2 n-18}$, $x^{\{3-6\}} z_{2 n-18}, x^{\{0-2\}} w^{\epsilon} \alpha^{k} z_{2 n}$, and $x^{\{3-6\}} z_{2 n}$ where $2 w^{\epsilon} \alpha^{k} z_{2 n-18}=x^{2} w^{\epsilon} \alpha^{k+1} z_{2 n}$. Furthermore, $\quad u z_{2 n-18}=x^{2} z_{2 n}, \quad u^{2} z_{2 n-18}=0=u z_{2 n}, \quad x^{2} \alpha^{k+1} z_{2 n-18}=\alpha_{3} \alpha^{k} z_{2 n}$, $x^{2} w \alpha^{k} z_{2 n-18}=\alpha_{1} \alpha^{k} z_{2 n}$, and $x^{6} z_{2 n-18}=\alpha_{2} z_{2 n}$. 
This follows automatically from the suspension isomorphism but we want to carefully write down the differentials and representations.

$$
\begin{gathered}
E^{1}=E^{2}=E^{3} \simeq v_{2}^{i} \alpha^{k} u^{n}, \quad 0 \leqslant i<8, \quad 0 \leqslant k \\
d^{3}\left(v_{2}^{6} \alpha^{k} v_{2}^{5 n} u^{n}\right)=\alpha^{k+1} v_{2}^{5 n} u^{n} \leftarrow z_{2 n} ; \\
d^{3}\left(v_{2}^{2} \alpha^{k} v_{2}^{5 n} u_{2 n} ;\right. \\
d^{3}\left(v_{2}^{6} \alpha^{k} v_{2}^{5 n+3} u^{n}\right)=v_{2}^{4} \alpha^{k+1} v_{2}^{5 n} u^{n+1} z_{2 n-18} ; \\
d^{3}\left(v_{2}^{2} \alpha^{k} v_{2}^{5 n+3} u^{n}\right)=v_{2}^{4} \alpha^{k+1} v_{2}^{5 n+3} u^{n} \leftarrow z_{2 n-18} ; \\
E^{4}=E^{5}=E^{6}=E^{7} \simeq v_{2}^{\{0,3,4,7\}} v_{2}^{5 n} u^{n} . \\
d^{7}\left(v_{2}^{4} v_{2}^{5 n} u^{n}\right)=v_{2}^{5 n} u^{n} \leftarrow \\
d_{2 n}\left(v_{2}^{7} v_{2}^{5 n} u^{n}\right)=v_{2}^{3} v_{2}^{5 n} u^{n} \leftarrow \\
z_{2 n-18} .
\end{gathered}
$$

\section{The spectral sequence for $\operatorname{ER}(2)^{*}\left(R P^{\infty} / R P^{16 K}\right)$}

We need $E R(2)^{*}\left(R P^{\infty} / R P^{16 K}\right)$ for our applications in this paper. It is essentially the same computation as for $E R(2)^{*}\left(R P^{\infty}\right)$ but the proof requires more care.

Theorem 11.1 (The Bockstein spectral sequence for $\left.E R(2)^{*}\left(R P^{\infty} / R P^{16 K}\right)\right)$.

$E^{1}=E(2)^{*}\left(R P^{\infty} / R P^{16 K}\right) \subset E(2)^{*}\left(R P^{\infty}\right)$ has 2-adic basis

$$
\begin{gathered}
v_{2}^{i} \alpha^{k} u^{j}, \quad 0 \leqslant i<8, \quad 0 \leqslant k, \quad 8 K<j \\
d^{1}\left(v_{2}^{2 s-5} \alpha^{k} u^{j}\right)=2 v_{2}^{2 s} \alpha^{k} u^{j} \equiv v_{2}^{2 s} \alpha^{k+1} u^{j+1}
\end{gathered}
$$

modulo higher powers of $u$.

$E^{2}=E^{3}$ is given by:

$$
\begin{aligned}
& v_{2}^{2 s} \alpha^{k} u^{8 K+1}, \quad v_{2}^{2 s} u^{j}, \quad 8 K+2 \leqslant j, \quad 0 \leqslant s<4, \quad 0 \leqslant k \\
& d^{3}\left(v_{2}^{4 s-2} \alpha^{k} u^{8 K+1}\right)=v_{2}^{4 s} \alpha^{k+1} u^{8 K+1} \text {, }
\end{aligned}
$$

and for $8 K+2 \leqslant j$

$$
d^{3}\left(v_{2}^{4 s-2} u^{j}\right)=v_{2}^{4 s} \alpha u^{j} \equiv v_{2}^{4 s} u^{j+2}
$$

modulo higher powers of $u$.

$E^{4}=E^{5}=E^{6}=E^{7}$ is given by

$$
\begin{array}{r}
v_{2}^{4} u^{8 K+\{1-3\}} \text { and } u^{8 K+\{1-3\}} \\
d^{7}\left(v_{2}^{4} u^{8 K+\{1-3\}}\right)=u^{8 K+\{1-3\}}
\end{array}
$$

There is an element $z_{16 K-16} \in E R(2)^{*}\left(R P^{\infty} / R P^{16 K}\right)$ that maps to $u^{8 K+1} \in$ $\operatorname{ER}(2)^{*}\left(R P^{\infty}\right)$

The $x^{1}$-torsion generators are given by:

$$
\alpha_{i} \alpha^{k} z_{16 K-16} u^{j}, \quad 0 \leqslant i<4, \quad 0 \leqslant k, \quad 0 \leqslant j
$$

where $\alpha_{0}=2$. 
The $x^{3}$-torsion generators are given by:

$$
w^{\epsilon} \alpha^{k} z_{16 K-16}, \quad \epsilon+k>0, \quad w z_{16 K-16} u^{j}, \quad 0<j, \text { and } z_{16 K-16} u^{j}, \quad 3 \leqslant j .
$$

The only $x^{7}$-torsion generators are

$$
z_{16 K-16} u^{\{0-2\}} .
$$

Proof. The $E^{1}$-term of the spectral sequence injects to the $E^{1}$-term for $E R(2)^{*}\left(R P^{\infty}\right)$ so $d^{1}$ is induced. The differential $d^{3}$ is a trickier issue. We look at the element in $E^{3}$ we have named $u^{8 K+1}$. If we have $d^{3}\left(u^{8 K+1}\right)=v_{2}^{2} z \neq 0$, then $v_{2}^{2} z$ must map to zero in $E^{3}$ for $E R(2)^{*}\left(R P^{\infty}\right)$. Since we had an injection on $E^{1}, v_{2}^{2} z$ must go to $2 y$ for some $y$. The only such elements are $v_{2}^{2} 2 \alpha^{k} u^{8 K} \equiv v_{2}^{2} \alpha^{k+1} u^{8 K+1}$ modulo higher powers of $u$. Now we use the map $R P^{16 K+2} / R P^{16 K} \rightarrow R P^{\infty} / R P^{16 K}$ where $u^{8 K+1}$ goes to $z_{16 K-16}$ in the spectral sequence. In the Bockstein spectral sequence for $E R(2)^{*}\left(R P^{16 K+2} / R P^{16 K}\right)$, $z_{16 K-16}$ has $d^{3}$ trivial but $v_{2}^{2} \alpha^{k+1} z_{16 K-16}$ is nonzero, so our $d^{3}$ must be zero on $u^{8 K+1}$. The differential $d^{3}$ then follows from $d^{3}\left(v_{2}^{2}\right)=\alpha v_{2}^{4}$. Likewise, our $d^{7}$ follows by comparison with $R P^{16 K+2} / R P^{16 K}$.

The same argument gives quite a different result when $m \neq 8 K$.

\section{The Bockstein spectral sequence for $\operatorname{ER}(2)^{*}\left(R P^{6}\right)$}

Before we proceed to $E R(2)^{*}\left(R P^{2 n}\right)$ we need to do the equivalent of starting an induction. This will be a little different from what we have done before and will show some of what is to come. We need just a simple fact about $E R(2)^{*}\left(R P^{6}\right)$.

Proposition 12.1. In $E R(2)^{*}\left(R P^{6}\right)$ the elements $u^{1-3}$ are $x^{7}$-torsion and $d^{7}$ takes $v_{2}^{4} u^{1-3}$ to them in the Bockstein spectral sequence.

Proof. We begin by computing $d^{1}$ in our spectral sequence where $E^{1}$ is represented by $v_{2}^{i} \alpha^{k} u^{j}$ for $1 \leqslant j \leqslant 3$. Our $E^{2}=E^{3}$-term is something new:

$$
v_{2}^{2 s} \alpha^{k} u, \quad v_{2}^{2 s} u^{\{2,3\}} \text {, and } v_{2}^{2 s+1} \alpha^{k} u^{3} \text {. }
$$

Comparing our spectral sequence with those for $R P^{6} / R P^{4}$ and $R P^{\infty}$ we can compute our $d^{3}$ to get $E^{4}=E^{5}$ :

$$
v_{2}^{\{0,4\}} u^{\{1-3\}} ; \quad v_{2}^{\{2,6\}} u^{\{2,3\}} ; \quad v_{2}^{\{3,7\}} u^{3} .
$$

For purely degree reasons, there are no $d^{5}$ differentials. Since $u$ must be a target for $d^{7}$, the $d^{7}$ differential is what we stated. The $d^{7}$ on the rest is solved by comparison again with $R P^{6} / R P^{4}$ but we don't need that in the statement of the theorem.

\section{The Bockstein spectral sequence for $\operatorname{ER}(2)^{*}\left(R P^{2 n}\right)$}

We want to compute the Bockstein spectral sequence for $E R(2)^{*}\left(R P^{2 n}\right)$. It isn't really that hard to do except that it breaks up into 8 distinct cases depending on $n$ modulo 8. For now we want to assume that $n>3$. Keep in mind that we have an even degree spectral sequence so all $d^{2 r}$ are zero because they are odd degree. We only have $d^{\{1,3,5,7\}}$ to consider. We have already computed $n=1$ and $n=3(n=2$ 
isn't hard). The differential $d^{1}$ does not depend on $n$. (Keep in mind that we always represent our groups with our 2-adic basis.)

$$
\begin{gathered}
E^{1} \simeq v_{2}^{i} \alpha^{k} u^{j}, \quad 0 \leqslant i<8, \quad 0 \leqslant k, \quad 0<j \leqslant n ; \\
d^{1}\left(v_{2}^{2 s-5} \alpha^{k} u^{j}\right)=2 v_{2}^{2 s} \alpha^{k} u^{j}
\end{gathered}
$$

for $j<n$. These elements represent the $x^{1}$-torsion elements $\alpha_{s} \alpha^{k} u^{j}, j<n$. We know that $2 \alpha^{k} u^{j} \equiv \alpha^{k+1} u^{j+1}$ modulo higher powers of $u$ so we have, for $E^{2}=E^{3}$ :

$$
v_{2}^{2 s} \alpha^{k} u, \quad v_{2}^{2 s} u^{j}, \quad v_{2}^{2 s+1} \alpha^{k} u^{n}, \quad 0 \leqslant k, \quad 1<j \leqslant n, \quad 0 \leqslant s<4 .
$$

We know that $\alpha^{k} u$ and $u^{j}$ are infinite cycles because they are in the image from $E R(2)^{*}\left(R P^{\infty}\right)$ so we can compute $d^{3}$ on the first two terms just using $d^{3}\left(v_{2}^{\{2,6\}}\right)=$ $\alpha v_{2}^{\{4,0\}}$. We use the fact that $E^{3}$ is a vector space over $\mathbb{Z} /(2)$. That reduces our relation to $0=\alpha u^{2}+_{F} u^{4}$. Modulo higher powers of $u$, this is just $\alpha u^{2} \equiv u^{4}$. So, modulo higher powers of $u$ we have:

$$
\begin{gathered}
d^{3}\left(v_{2}^{\{6,2\}} \alpha^{k} u\right)=v_{2}^{\{0,4\}} \alpha^{k+1} u ; \\
d^{3}\left(v_{2}^{\{6,2\}} u^{j}\right)=v_{2}^{\{0,4\}} \alpha u^{j} \equiv v_{2}^{\{0,4\}} u^{j+2}, \quad 1<j \leqslant n-2 .
\end{gathered}
$$

The $E^{1}$ of the Bockstein spectral sequence for $E R(2)^{*}\left(R P^{2 n} / R P^{2 n-2}\right)$, i.e. $E(2)^{*}$ $\left(R P^{2 n} / R P^{2 n-2}\right)$, injects to that for $E R(2)^{*}\left(R P^{2 n}\right)$. The map is given by:

$$
\begin{gathered}
z_{2 n}=z_{16 K+2 j} \longrightarrow v_{2}^{5 j} u^{8 K+j} \\
z_{2 n-18}=z_{16 K+2 j-18} \longrightarrow v_{2}^{5 j+3} u^{8 K+j},
\end{gathered}
$$

where $0<j \leqslant 8$. In particular, when $j=3,4,7$ or 8 , either $v_{2}^{7}$ or $v_{2}^{3}$ times $u^{8 K+j}$ is in the image and can therefore have no differential. The usual $d^{3}\left(v_{2}^{2}\right)=\alpha v_{2}^{4}$ determines the differentials:

$$
d^{3}\left(v_{2}^{\{2,6\}} v_{2}^{7} u^{8 K+j}\right)=v_{2}^{\{4,0\}} v_{2}^{7} \alpha u^{8 K+j} .
$$

Similarly when $j=1,2,5$ or 6 , we have $v_{2}^{5}$ or $v_{2}$ times $u^{8 K+j}$ in the image and we get

$$
d^{3}\left(v_{2}^{\{2,6\}} v_{2}^{5} u^{8 K+j}\right)=v_{2}^{\{4,0\}} v_{2}^{5} \alpha u^{8 K+j} .
$$

Combining all of our computations for $d^{3}$ we have $E^{4}=E^{5}$

$$
v_{2}^{\{0,4\}} u^{\{1-3\}} ; \quad v_{2}^{\{6,2\}} u^{\{n-1, n\}} ; \quad v_{2}^{\{2 s+1,2 s+5\}} u^{n}
$$

where $s=0$ if $n \equiv 1,2,5$ or $6 \bmod 8$, and $s=1$ if $n \equiv 3,4,7$ or $8 \bmod 8$.

We have computed $E R(2)^{*}\left(R P^{6}\right)$ and shown that the elements $u^{\{1-3\}}$ are all $x^{7}$ torsion and $d^{7}\left(v_{2}^{4} u^{\{1-3\}}\right)=u^{\{1-3\}}$. By naturality, the elements $u^{\{1-3\}} \in E R(2)^{*}\left(R P^{2 n}\right)$ must also be $x^{7}$ torsion with the same differential. The only elements we have left to worry about in our spectral sequence are:

$$
v_{2}^{\{6,2\}} u^{\{n-1, n\}} ; \quad v_{2}^{\{2 s+1,2 s+5\}} u^{n}
$$

where $s=0$ or 1 as above. 
We collect what we know so far in the following preliminary result:

Theorem 13.2. Let $n>3$. The Bockstein spectral sequence for $E R(2)^{*}\left(R P^{2 n}\right)$ begins as follows (with differentials modulo higher powers of $u$ ):

$E^{1}=E(2)^{*}\left(R P^{2 n}\right)$ is represented by

$$
\begin{gathered}
v_{2}^{i} \alpha^{k} u^{j}, \quad 0 \leqslant i<8, \quad 0 \leqslant k, \quad 0<j \leqslant n ; \\
d^{1}\left(v_{2}^{2 s-5} \alpha^{k} u^{j}\right)=2 v_{2}^{2 s} \alpha^{k} u^{j} \equiv v_{2}^{2 s} \alpha^{k+1} u^{j+1}, \quad j<n .
\end{gathered}
$$

$E^{2}=E^{3}$ is given by

$$
\begin{gathered}
v_{2}^{2 s} \alpha^{k} u, \quad 0 \leqslant k ; \quad v_{2}^{2 s} u^{j}, \quad 1<j \leqslant n ; \quad v_{2}^{2 s+1} \alpha^{k} u^{n}, \quad 0 \leqslant k ; \\
d^{3}\left(v_{2}^{\{6,2\}} \alpha^{k} u\right)=v_{2}^{\{0,4\}} \alpha^{k+1} u ; \\
d^{3}\left(v_{2}^{\{6,2\}} u^{j}\right)=v_{2}^{\{0,4\}} \alpha u^{j} \equiv v_{2}^{\{0,4\}} u^{j+2}, \quad 1<j \leqslant n-2 ; \\
d^{3}\left(v_{2}^{\{2 s+1,2 s+5\}} \alpha^{k} u^{n}\right)=v_{2}^{\{2 s+3,2 s+7\}} \alpha^{k+1} u^{n} ;
\end{gathered}
$$

where $s=0$ for $n \equiv 3,4,7$ and 8 modulo 8 and $s=1$ for $n \equiv 1,2,5$ and 6 modulo 8 . $E^{4}=E^{5}$ is given by

$$
v_{2}^{\{0,4\}} u^{\{1-3\}} ; \quad v_{2}^{\{6,2\}} u^{\{n-1, n\}} ; \quad v_{2}^{\{2 s+1,2 s+5\}} u^{n} ;
$$

where $s=0$ if $n \equiv 1,2,5$ or $6 \bmod 8$, and $s=1$ if $n \equiv 3,4,7$ or $8 \bmod 8$.

$$
d^{7}\left(v_{2}^{4} u^{\{1-3\}}\right)=u^{\{1-3\}} .
$$

The only remaining undetermined part of the Bockstein spectral sequence is in $E^{5}$ :

$$
v_{2}^{\{6,2\}} u^{\{n-1, n\}} ; \quad v_{2}^{\{2 s+1,2 s+5\}} u^{n}
$$

where $s$ is 0 or 1 as above.

We now have to start working our way through the 8 cases. There can be significant variation on what happens. We only have 6 elements here in our basis and we must kill them all off with $d^{5}$ and $d^{7}$. For purely degree reasons, if there is a $d^{5}$ it must be $d^{5}\left(v_{2}^{\{6,2\}} u^{n-1}\right)=v_{2}^{\{5,1\}} u^{n}$. Of course, if those last elements aren't there, $d^{5}$ must be zero.

We collect the remaining differentials for all 8 cases in one place:

Theorem 13.3. The remaining differentials for the Bockstein spectral sequence for $\operatorname{ER}(2)^{*}\left(R P^{2 n}\right), n>3$, together with a little of the map

$$
q^{*}: E R(2)^{*}\left(R P^{2 n} / R P^{2 n-2}\right) \rightarrow E R(2)^{*}\left(R P^{2 n}\right)
$$

are as follows: 
$E R(2)^{*}\left(R P^{16 K+2}\right)$

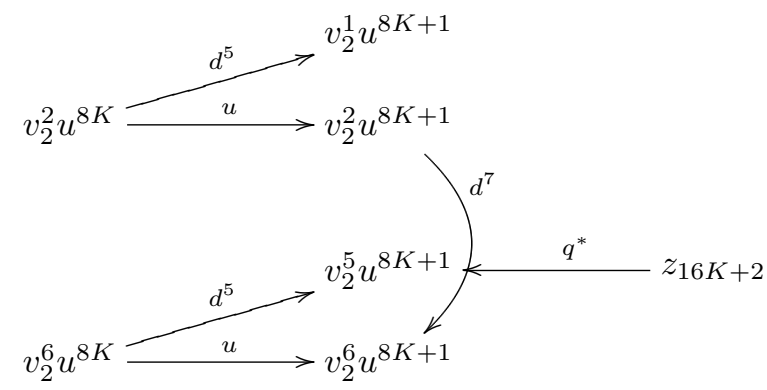

$E R(2)^{*}\left(R P^{16 K+4}\right)$

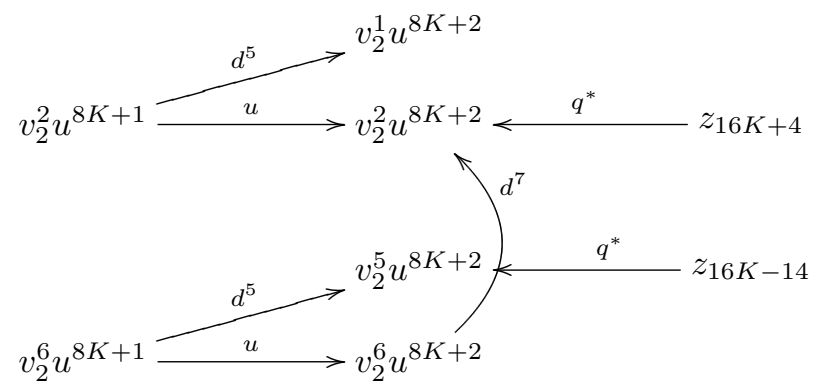

$E R(2)^{*}\left(R P^{16 K+6}\right)$

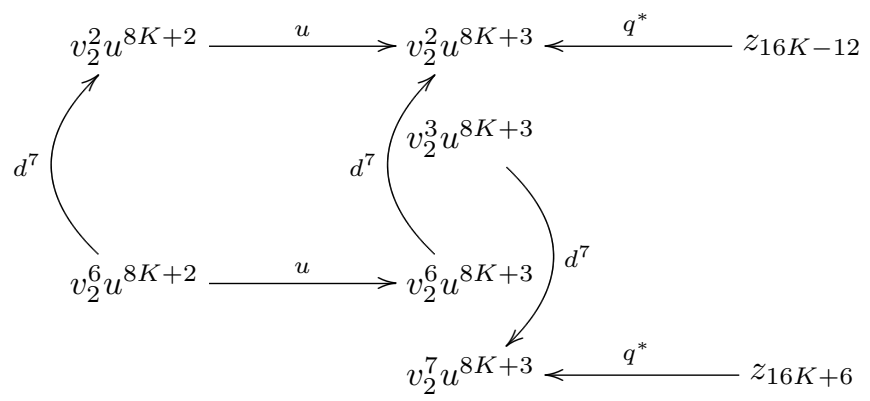

$E R(2)^{*}\left(R P^{16 K+8}\right)$

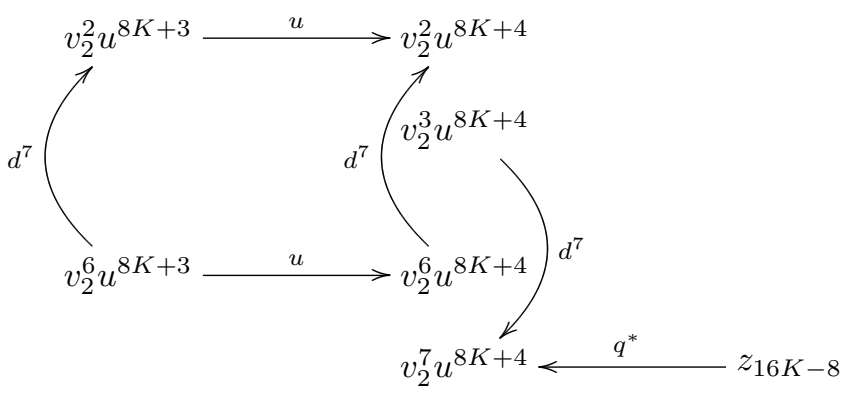


$\operatorname{ER}(2)^{*}\left(R P^{16 K+10}\right)$

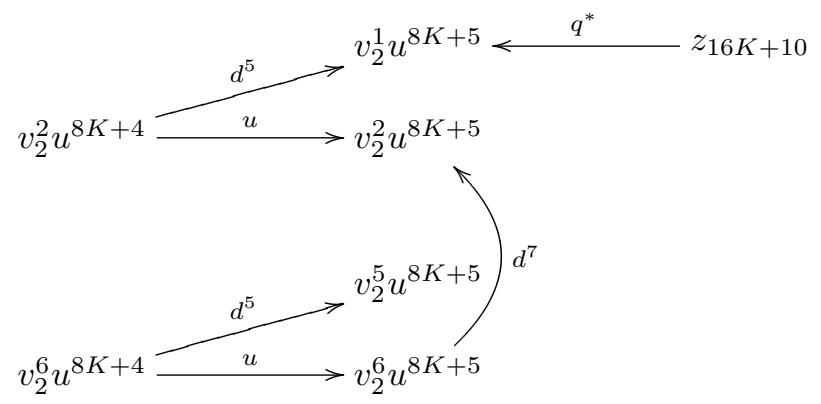

$\operatorname{ER}(2)^{*}\left(R P^{16 K+12}\right)$
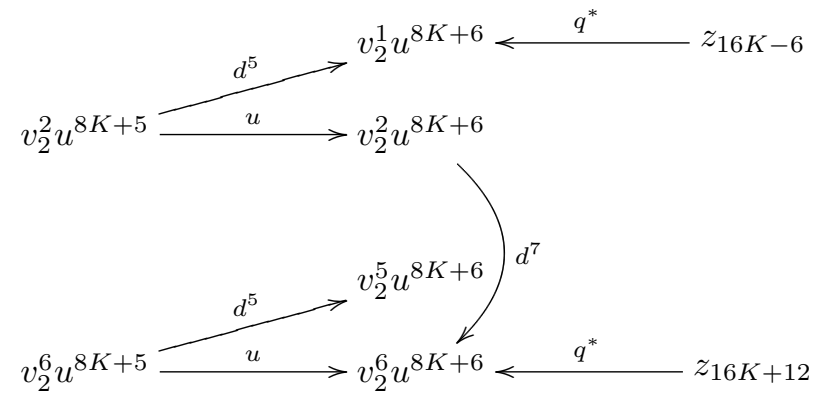

$\operatorname{ER}(2)^{*}\left(R P^{16 K+14}\right)$

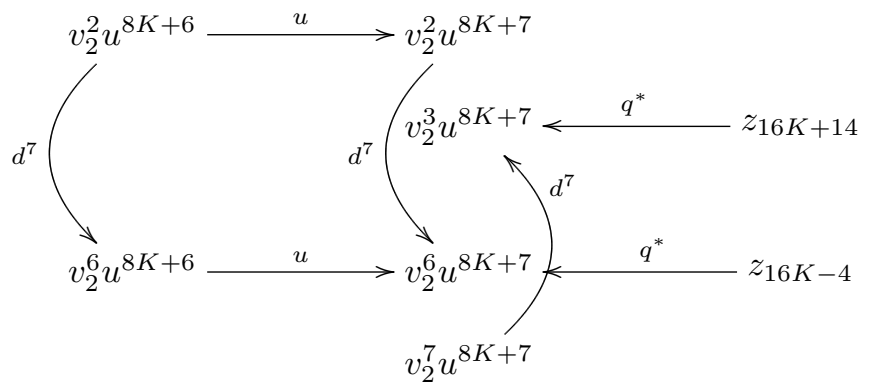

$E R(2)^{*}\left(R P^{16 K+16}\right)$

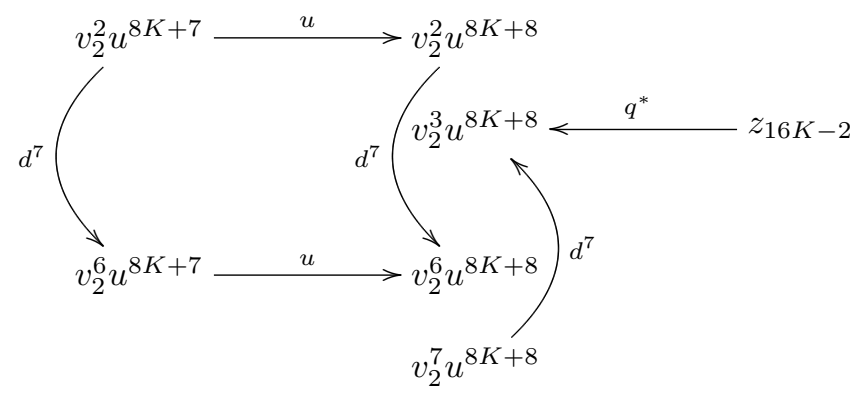


Proof. As already discussed, for degree reasons, there can be no $d^{5}$ for the Bockstein spectral sequence for $E R(2)^{*}\left(R P^{2 n}\right)$ when $n \equiv 3,4,7$ or $8 \bmod 8$. The two $d^{7}$ differentials for $n \equiv 3$ and $7 \bmod 8$ follow from the map $R P^{2 n} \rightarrow R P^{2 n} / R P^{2 n-2}$. One of the $d^{7}$ differentials for $n \equiv 4$ and 8 mod 8 follows from the map $R P^{2 n} \rightarrow R P^{2 n} / R P^{2 n-2}$ and the other follows from the map $R P^{2 n-2} \rightarrow R P^{2 n}$. This completes the four cases $n \equiv 3,4,7$ and $8 \bmod 8$.

The other four cases all have a non-trivial $d^{5}$.

We begin by looking at the $n \equiv 2 \bmod 8$ case. The map to the $n \equiv 3 \bmod 8$ case takes care of $d^{7}\left(v_{2}^{6} u^{8 K+2}\right)=d^{7}\left(v_{2}^{2} u^{8 K+2}\right)$. If there is no $d^{5}$, it would also give the $d^{7}$ on $v_{2}^{6} u^{8 K+1}$ and we would have a generator, represented by $v_{2}^{2} u^{8 K+1}$, that was not in the image of the $n \equiv 3 \bmod 8$ case. We now use the cofibration $R P^{2 n-2} \rightarrow$ $R P^{2 n} \rightarrow R P^{2 n} / R P^{2 n-2}$ where $n \equiv 3 \bmod 8$. We have a complete description of $E R(2)^{*}\left(R P^{2 n} / R P^{2 n-2}\right)$. All of the elements associated with $z_{2 n}$ inject, i.e. $x^{\{0-6\}} z_{2 n}$ and $x^{\left\{0^{-2}\right\}} w^{\epsilon} \alpha^{k} z_{2 n}$. We also have $x^{\{0-6\}} z_{2 n-18}$ and $w^{\epsilon} \alpha^{k} z_{2 n-18}$ injecting. The only possible elements left for the kernel are $x^{\{1,2\}} w^{\epsilon} \alpha^{k} z_{2 n-18}$, where $\epsilon+k>0$. Thus the boundary on the element represented by $v_{2}^{2} u^{8 K+1}$ must hit one of these elements. The boundary homomorphism increases degree by 1 so, modulo 8 , the degree of the image is -3 . However, the degrees, modulo 8, of the elements $x^{\{1,2\}} w^{\epsilon} \alpha^{k} z_{2 n-18}$ are -5 and -6 (remember, $n \equiv 3 \bmod 8$ here). There must be a $d^{5}$ to prevent this impossibility. A similar argument works for $n \equiv 6 \bmod 8$ comparing it with $n \equiv 7 \bmod 8$.

We work on the $n \equiv 1 \bmod 8$ case now using the cofibration $R P^{2 n-2} \rightarrow R P^{2 n} \rightarrow$ $R P^{2 n} / R P^{2 n-2}$ for $n \equiv 2 \bmod 8$. Here, all of the elements associated with $z_{2 n-18}$ inject, with the possible exception of $x^{\{5,6\}} z_{2 n-18}$. The other possible elements in the kernel are $x^{\{1,2\}} w^{\epsilon} \alpha^{k} z_{2 n}, \epsilon+k>0$. If there is no $d^{5}$, then $d^{7}\left(v_{2}^{2} u^{8 K}\right)=v_{2}^{6} u^{8 K}$ is determined by comparison with the $n \equiv 0 \bmod 8$ case. The element representing $v_{2}^{6} u^{8 K}$ is not in the image and so must have boundary non-trivial in the above cofibration for $n \equiv 2 \bmod 8$. The degree of the boundary of this element is $16 K-35$. Using the $n \equiv 2 \bmod 8$ cofibration the degrees of $x^{\{1,2\}} w^{\epsilon} \alpha^{k} z_{2 n} \bmod 8$ are -5 and -6 . The degrees of $x^{\{5,6\}} z_{2 n-18}$ are $-5 \times 17+16 K+4-18=16 K-3$ and $16 K-4$. There is nowhere for our element to go so there must be a $d^{5}$.

We still have to deal with the $d^{7}$ because it is not induced by any of our maps. One of $v_{2}^{\{2,6\}} u^{8 K+1}$ must have a non-trivial boundary homomorphism on it. The degree of the boundary image will be $16 K-16-12+1=16 K-27$ (for $v_{2}^{2} u^{8 K+1}$ ) or $16 K-3$ (for $\left.v_{2}^{6} u^{8 K+1}\right)$. Thus we must have $d^{7}\left(v_{2}^{2} u^{8 K+1}\right)=v_{2}^{6} u^{8 K+1}$ and the boundary of $v_{2}^{6} u^{8 K+1}$ must hit $x^{5} z_{2 n-18}$, a fact sure to be useful sometime.

A similar argument works for $n \equiv 5 \bmod 8$ comparing it to $n \equiv 6 \bmod 8$.

For our applications, what we really need to know is $E R(2)^{16 *}\left(R P^{2 n}\right)$ and how these elements sit in $E R(2)^{*}\left(R P^{2 n}\right)$. The simple version of this is stated in the Introduction as Theorem 1.6.

Theorem 13.4. For all $n$ there is a short exact sequence:

$$
E R(2)^{16 *}\left(R P^{2 n-2}\right) \leftarrow E R(2)^{16 *}\left(R P^{2 n}\right) \leftarrow \quad E R(2)^{16 *}\left(R P^{2 n} / R P^{2 n-2}\right) .
$$

We have elements $\alpha^{k} u^{j} \in E R(2)^{16 *}\left(R P^{2 n}\right), 0 \leqslant k, 0<j \leqslant n$ that reduce to elements 
THE SECOND REAL JOHNSON-WILSON THEORY AND NONIMMERSIONS OF $R P^{n} \quad 249$

of the same name in $E(2)^{16 *}\left(R P^{2 n}\right)$. Depending on $n$ modulo 8 there are other elements in $E R(2)^{16 *}\left(R P^{2 n}\right)$.

For $n=8 K+8$ and $8 K+7$ there are no other elements and $u^{n+1}=0$.

For $n=8 K+6$ there is an $x^{5}$-torsion element, $z_{16 K-30}$, that reduces to $v_{2}^{5} u^{8 K+6}$ in the Bockstein spectral sequence such that

$$
x^{2} \alpha^{k} z_{16 K-30}=\alpha^{k} u^{8 K+7}
$$

and $u^{n+2}=0$.

For $n=8 K+5$ there is an $x^{5}$-torsion element, $z_{16 K-14}$, that reduces to $v_{2}^{5} u^{8 K+5}$ in the Bockstein spectral sequence such that

$$
x^{2} \alpha^{k} z_{16 K-14}=\alpha^{k} u^{8 K+6}
$$

and an $x^{7}$-torsion element, $z_{16 K+4}$ that reduces to $v_{2}^{2} u^{8 K+5}$ in the Bockstein spectral sequence such that

$$
x^{2} u z_{16 K-14}=x^{4} z_{16 K+4}=u^{8 K+7}
$$

and $u^{n+3}=0$.

For $n=8 K+4$ there are $x^{7}$-torsion elements, $z_{16 K-12}$, and $z_{16 K-10}$ that reduce to $v_{2}^{2} u^{8 K+3}$ and $v_{2}^{7} u^{8 K+4}$ respectively in the Bockstein spectral sequence such that

$$
\begin{gathered}
x^{4} z_{16 K-12}=u^{8 K+5}, \\
x^{4} u z_{16 K-12}=u^{8 K+6},
\end{gathered}
$$

and

$$
x^{4} u^{2} z_{16 K-12}=x^{6} z_{16 K-10}=u^{8 K+7}
$$

and $u^{n+4}=0$.

For $n=8 K+3$ there are $x^{7}$-torsion elements, $z_{16 K+4}$, and $z_{16 K-42}$ that reduce to $v_{2}^{2} u^{8 K+2}$ and $v_{2}^{7} u^{8 K+3}$ respectively in the Bockstein spectral sequence such that

$$
\begin{gathered}
x^{4} z_{16 K+4}=u^{8 K+4}, \\
x^{4} u z_{16 K+4}=u^{8 K+5},
\end{gathered}
$$

and

$$
x^{4} u^{2} z_{16 K+4}=x^{6} z_{16 K-42}=u^{8 K+6}
$$

and $u^{n+4}=0$.

For $n=8 K+2$ there is an $x^{5}$-torsion element, $z_{16 K-14}$, that reduces to $v_{2}^{5} u^{8 K+2}$ in the Bockstein spectral sequence such that

$$
x^{2} \alpha^{k} z_{16 K-14}=\alpha^{k} u^{8 K+3}
$$

and an $x^{7}$-torsion element, $z_{16 K+4}$ that reduces to $v_{2}^{2} u^{8 K+2}$ in the Bockstein spectral sequence such that

$$
x^{2} u z_{16 K-14}=x^{4} z_{16 K+4}=u^{8 K+4}
$$

and $u^{n+3}=0$. 
For $n=8 K+1$ there is an $x^{5}$-torsion element, $z_{16 K+2}$, that reduces to $v_{2}^{5} u^{8 K+1}$ in the Bockstein spectral sequence such that

$$
x^{2} \alpha^{k} z_{16 K+2}=\alpha^{k} u^{8 K+2}
$$

and $u^{n+2}=0$.

Proof. We have computed the Bockstein spectral sequence for all of the spaces $R P^{2 n-2}, R P^{2 n}$, and $R P^{2 n} / R P^{2 n-2}$. From this we can just read off the elements in degree $16 *$. In every case the $x^{1}$-torsion elements $\alpha_{0} \alpha^{k} u^{j}$ for $j<n-1$ correspond using the map induced by $R P^{2 n-2} \rightarrow R P^{2 n}$. Likewise for the elements $\alpha^{k} u, u^{\{1-3\}}$, and $u^{j}, j<n$ so we will ignore these elements.

First note that $\alpha_{0} \alpha^{k} u^{n-1}=2 \alpha^{k} u^{n-1}=\alpha^{k+1} u^{n}$.

For $n \equiv 8 \bmod 8$, there is nothing else in $E R(2)^{16 *}\left(R P^{2 n-2}\right)$. All that is left of $(13.5)$ is $\alpha^{k} z_{2 n} \rightarrow \alpha^{k} u^{n}$.

From Theorem 10.1 we know $u z_{2 n}=0$ and, from above, this maps to $u^{n+1}$ so it is zero. Because $z_{2 n}$ maps to $u^{n}$, we see that $u^{8 K+8}=0$ for $n=8 K+7,8 K+6$, $8 K+5$, and $8 K+4$.

For $n \equiv 7 \bmod 8, E R(2)^{16 *}\left(R P^{2 n} / R P^{2 n-2}\right)=0$. We must have that $\alpha^{k} u^{n} \rightarrow$ $x^{2} \alpha^{k} u^{n-1} v_{2}^{5}$. (Technically, we need to worry that perhaps $u^{n}$ goes to $x^{2} \alpha^{3 k} u^{n-1} v_{2}^{5}$ for some $k$. If this is the case, then the boundary homomorphism on $x^{2} u^{n-1} v_{2}^{5}$ must be non-trivial but we can check that there is nowhere for it to go. Consequently we will ignore this kind of possibility in the rest of this proof.)

For $n \equiv 6 \bmod 8$, things are a little more complicated. The only elements in $E R(2)^{16 *}\left(R P^{2 n} / R P^{2 n-2}\right)$ are $x^{2} w \alpha^{k} z_{2 n-18}$ and we can compute directly that they go to $x^{2} \alpha^{k+1} u^{n} v_{2}^{5}$. The element $\alpha^{k} u^{n}$ must go to $x^{2} \alpha^{k} u^{n-1} v_{2}^{5}$. The only possibility left is for $x^{2} u^{n} v_{2}^{5}$ to go to $x^{4} u^{n-1} v_{2}^{2}$. Recall from above that this last element is $u^{n+1}$.

For $n \equiv 5 \bmod 8$, we compute the map to $E R(2)^{16 *}\left(R P^{2 n}\right)$ directly and we have

$$
\begin{gathered}
w \alpha^{k} z_{2 n-18} \longrightarrow \alpha^{k+1} u^{n} ; \\
x^{2} w \alpha^{k} z_{2 n} \longrightarrow x^{2} \alpha^{k+1} u^{n} v_{2}^{5} .
\end{gathered}
$$

Keep in mind that this last represents $\alpha^{k+1} u^{n+1}$. We then have $u^{n+1}=x^{2} u^{n} v_{2}^{5}$ maps to $x^{4} u^{n-1} v_{2}^{2}$. We must have $u^{n}$ map to $x^{4} u^{n-2} v_{2}^{2}$ and $x^{4} u^{n} v_{2}^{2}$ (which represents $u^{n+2}$ ) map to $x^{6} u^{n-1} v_{2}^{7}$.

For $n \equiv 4 \bmod 8$ we compute $x^{6} z_{2 n-18} \rightarrow x^{6} u^{n} v_{2}^{7}=u^{n+3}$ and $w \alpha^{k} z_{2 n} \rightarrow \alpha^{k+1} u^{n}$. That leaves $u^{n} \rightarrow x^{4} u^{n-2} v_{2}^{2}, x^{4} u^{n-1} v_{2}^{2}=u^{n+1} \rightarrow x^{4} u^{n-1} v_{2}^{2}$, and $x^{4} u^{n} v_{2}^{2}=u^{n+2} \rightarrow$ $x^{6} u^{n-1} v_{2}^{7}$.

Because $u^{n+3}$ is in the image above, it must go to zero for the next case below.

For $n \equiv 3 \bmod 8$ we compute $x^{4} z_{2 n-18} \rightarrow x^{4} u^{n} v_{2}^{2}=u^{n+2}$ and $x^{6} z_{2 n} \rightarrow x^{6} u^{n} v_{2}^{7}=$ $u^{n+3}$. That leaves $x^{4} u^{n-1} v_{2}^{2}=u^{n+1} \rightarrow x^{4} u^{n-1} v_{2}^{2}$ and $\alpha^{k} u^{n} \rightarrow x^{2} \alpha^{k} u^{n-1} v_{2}^{5}$.

Because both $u^{n+2}$ and $u^{n+3}$ are in the image above, they must both go to zero in the next case, which gives us that $u^{8 K+5}=0$ for $n=8 K+2$.

For $n \equiv 2 \bmod 8$ we compute $x^{2} \alpha^{k} z_{2 n-18} \rightarrow x^{2} \alpha^{k} u^{n} v_{2}^{5}=\alpha^{k} u^{n+1}$ and $x^{4} z_{2 n} \rightarrow$ $x^{4} u^{n} v_{2}^{2}=u^{n+2}$. All that is left is $\alpha^{k} u^{n} \rightarrow x^{2} \alpha^{k} u^{n-1} v_{2}^{5}$.

Because both $u^{n+1}$ and $u^{n+2}$ are in the image above, they must both go to zero in the next case, which gives us that $u^{8 K+3}=0$ for $n=8 K+1$.

The $n \equiv 1 \bmod 8$ case is simple again with $\alpha^{k} z_{2 n-18} \rightarrow \alpha^{k} u^{n}$ and $x^{2} \alpha^{k} z_{2 n} \rightarrow$ $x^{2} \alpha^{k} u^{n} v_{2}^{5}=\alpha^{k} u^{n+1}$. 


\section{Beginning with products}

For use with our Bockstein spectral sequence we need descriptions of $E(2)^{*}(-)$ for various products. We always use reduced cohomology. We start with a result proven by modifying techniques of [JW85]:

Theorem $14.1([\mathbf{G W}])$. Let $m<n$; then

$$
B P^{*}\left(R P^{2 m} \wedge R P^{2 n}\right) \simeq B P^{*}\left(R P^{2 m}\right) \otimes_{B P^{*}} B P^{*}\left(R P^{2 n}\right) \oplus \Sigma^{2 n-1} B P^{*}\left(R P^{2 m}\right) .
$$

Remark 14.2. It is important to note, because we use it later, that this is natural in the obvious way for the $R P^{2 m}$ when $m<n$.

It is enough to prove this using $B P\langle 2\rangle$, where $B P\langle 2\rangle^{*} \simeq \mathbb{Z}_{(2)}\left[v_{1}, v_{2}\right]$, because $v_{2}$ multiplication is injective and so it determines the Brown-Peterson cohomology. We can now invert $v_{2}$ to get $E(2)^{*}(-)$ and the same theorem holds. Because there is no $v_{2^{-}}$ torsion, $B P\langle 2\rangle^{*}\left(R P^{2 m} \wedge R P^{2 n}\right)$ injects into $E(2)^{*}\left(R P^{2 m} \wedge R P^{2 n}\right)$. This is important because we rely on Don Davis's computations. He does his in $B P\langle 2\rangle^{*}(-)$ but this shows they could just as well have been done in $E(2)^{*}(-)$.

We do not use the standard notation because we need to be compatible with $\operatorname{ER}(2)^{*}(-)$. Above, the bottom class in the suspension is $\Sigma^{2 n-1} x_{2}$. We shift this using the unit $v_{2}^{3}$ raised to the $n$-th power, i.e. we shift the suspension down by $-18 n$ so our bottom class is now $\Sigma^{-16 n-1} x_{2}$ but we also replace $x_{2}$ with our $u=v_{2}^{3} x_{2}$. Our bottom class is now in degree $-16 n-1+2-18=-16 n-17$. We give it the name $z_{-16 n-17}$. The result for our 48-periodic theory that we use is as follows where we also include the more detailed description from $[\mathbf{G W}]$. Much of this is well known.

Theorem 14.3. Let $m<n$, then

$$
E(2)^{*}\left(R P^{2 m} \wedge R P^{2 n}\right) \simeq E(2)^{*}\left(R P^{2 m}\right) \otimes_{E(2)^{*}} E(2)^{*}\left(R P^{2 n}\right) \oplus \Sigma^{-16 n-1} E(2)^{*}\left(R P^{2 m}\right)
$$

has a 2-adic basis

$$
\begin{gathered}
v_{2}^{s} \alpha^{k} u_{1}^{i} u_{2}, \quad 0 \leqslant k, \quad 0<i \leqslant m, \quad 0 \leqslant s<8 \\
v_{2}^{s} u_{1}^{i} u_{2}^{j}, \quad 0<i \leqslant m, \quad 1<j \leqslant n, \quad 0 \leqslant s<8
\end{gathered}
$$

and

$$
\begin{gathered}
v_{2}^{s} \alpha^{k} u^{j} z_{-16 n-17}, \quad 0 \leqslant k, \quad 0 \leqslant j<m, \quad 0 \leqslant s<8 . \\
E(2)^{*}\left(R P^{2 n} \wedge R P^{\infty}\right) \simeq E(2)^{*}\left(R P^{2 n}\right) \otimes_{E(2)^{*}} E(2)^{*}\left(R P^{\infty}\right)
\end{gathered}
$$

has a 2-adic basis

$$
v_{2}^{s} \alpha^{k} u_{1}^{i} u_{2}, \quad 0 \leqslant k, \quad 0<i \leqslant n, \quad 0 \leqslant s<8
$$

and

$$
\begin{gathered}
v_{2}^{s} u_{1}^{i} u_{2}^{j}, \quad 0<i \leqslant n, \quad 1<j, \quad 0 \leqslant s<8 \\
E(2)^{*}\left(R P^{2 n} \wedge R P^{\infty} / R P^{2 m}\right) \simeq E(2)^{*}\left(R P^{2 n}\right) \otimes_{E(2)^{*}} E(2)^{*}\left(R P^{\infty} / R P^{2 m}\right)
\end{gathered}
$$


has a 2-adic basis

$$
\begin{gathered}
v_{2}^{s} \alpha^{k} u_{1}^{i} u_{2}^{m+1}, \quad 0 \leqslant k, \quad 0<i \leqslant n, \quad 0 \leqslant s<8 \\
v_{2}^{s} u_{1}^{i} u_{2}^{j}, \quad 0<i \leqslant n, \quad m+1<j, \quad 0 \leqslant s<8
\end{gathered}
$$

\section{A review of our relation}

We need a bit more detail about our relation

$$
0=[2](u)=\sum_{s \geqslant 0} a_{s} u^{s+1}=2 u+_{F} \alpha u^{2}+_{F} u^{4}
$$

The degree of our $a_{s}$ is $16 s$ and the degree of the relation is -16 .

\section{Lemma 15.1.}

$$
0=2 u+_{F} \alpha u^{2}+_{F} u^{4}=2 u+\alpha u^{2}+u^{4}+2 u^{3} z_{a}(u)+\alpha u^{6} z_{b}(u) .
$$

Proof. The proof follows immediately from the fact that $F(y, 0)=y$. The $z_{a}(u)$ and $z_{b}(u)$ are power series in $u$ and are not determined uniquely because many elements are divisible by both 2 and $\alpha$.

Definition 15.2. We need a filtration on our elements $\alpha^{k} u_{1}^{i} u_{2}$ and $u_{1}^{i} u_{2}^{j}$ in the tensor product part of our description of $E(2)^{*}\left(R P^{2 m} \wedge R P^{2 n}\right)$. We say $u_{1}^{a} u_{2}^{b}$ is of higher filtration than $u_{1}^{i} u_{2}^{j}$ if $a+b>i+j$ or, if $a+b=i+j$ and we have $a>i$.

Remark 15.3 (The Algorithm). We use no elements with a 2 or an $\alpha u_{2}^{2}$ in our tensor product description of $E(2)^{*}\left(R P^{2 m} \wedge R P^{2 n}\right)$ with $m<n$. We need an algorithm that shows how any element can be reduced to those in our description, i.e. $\alpha^{k} u_{1}^{i} u_{2}$ and $u_{1}^{i} u_{2}^{j}, j>1$. It is enough if our algorithm increases filtration as that will eventually lead to terms in our description. If we have a 2 , we use our relation

$$
2 u_{1}^{i} u_{2}^{j}=\left(2 u_{1}\right) u_{1}^{i-1} u_{2}^{j}=-\left(\sum_{k>0} a_{k} u_{1}^{k+1}\right) u_{1}^{i-1} u_{2}^{j} .
$$

All of these terms have higher filtration. If 2 does not divide and if $j=1$, then we are done. So, we are left with the case where $\alpha u_{2}^{2}$ divides our element. In this case, modulo higher filtrations, we have:

$$
\alpha u_{1}^{i} u_{2}^{j}=u_{1}^{i} u_{2}^{j-2}\left(\alpha u_{2}^{2}\right) \equiv u_{1}^{i} u_{2}^{j-2}\left(-2 u_{2}\right)=-2 u_{1}^{i} u_{2}^{j-1} .
$$

and we use the first reduction on this to get, modulo higher filtration, $\alpha u_{1}^{i+1} u_{2}^{j-1}$. Even this term is of higher filtration than we need. If neither 2 nor $\alpha$ is present, then we are done. However, there is one last step. Since we are using our 2-adic representation for everything, we only want 0 and 1 for coefficients. Whenever we have $-z$, we can replace it by $z-2 z$ and use the algorithm on $-2 z$. This shows that $-z \equiv z$ modulo higher filtration.

The algorithm ends after a finite number of steps when the power of $u_{1}$ is greater than $m$, the power of $u_{2}$ is greater than $n$, or the power of $u_{2}=1$ and there are no more 2's left. 
Lemma 15.4. There is an element $z$ with filtration greater than $u_{1} u_{2}^{2}$ such that

$$
2\left(u_{1} u_{2}^{2}+z\right) \equiv u_{1}^{2} u_{2}^{4}
$$

modulo filtrations higher than that of $u_{1}^{2} u_{2}^{4}$.

Proof. We compute with

$$
\begin{aligned}
& 2\left(u_{1} u_{2}^{2}-u_{1}^{2} u_{2}-u_{1}^{2} u_{2}^{3} z_{a}\left(u_{2}\right)+u_{1}^{3} z_{a}\left(u_{1}\right) u_{2}^{2}\right)= \\
& \quad-\left(\alpha+2 u_{1} z_{a}\left(u_{1}\right)+u_{1}^{2}+\alpha u_{1}^{4} z_{b}\left(u_{1}\right)\right) u_{1}^{2} u_{2}^{2} \\
& \quad+\left(\alpha+2 u_{1} z_{a}\left(u_{1}\right)+u_{1}^{2}+\alpha u_{1}^{4} z_{b}\left(u_{1}\right)\right) u_{1}^{3} u_{2} \\
& -2 u_{1}^{2} u_{2}^{3} z_{a}\left(u_{2}\right)+2 u_{1}^{3} z_{a}\left(u_{1}\right) u_{2}^{2} .
\end{aligned}
$$

The very first term, $-\alpha u_{1}^{2} u_{2}^{2}$, is, using the algorithm and ignoring higher terms:

$$
-\alpha u_{1}^{3} u_{2}-u_{1}^{5} u_{2}-2 u_{1}^{4} z_{a}\left(u_{1}\right) u_{2}+u_{1}^{2} u_{2}^{4}+2 u_{1}^{2} u_{2}^{3} z_{a}\left(u_{2}\right)
$$

Most terms now cancel out and we are left with, modulo the higher filtration terms, $u_{1}^{2} u_{2}^{4}$.

We are getting nearer to what we really need.

Lemma 15.5. For $u_{1}^{i} u_{2}^{j}$ with $j>1$ there is a $z$ in $E(2)^{*}\left(R P^{2 m} \wedge R P^{2 n}\right)$ with $m<n$ having higher filtration than $u_{1}^{i} u_{2}^{j}$ such that

$$
2\left(u_{1}^{i} u_{2}^{j}+z\right) \equiv u_{1}^{i+1} u_{2}^{j+2}
$$

modulo the terms $\alpha^{k} u_{1}^{c} u_{2}$ with $c \geqslant i+j+2, u_{1}^{c} u_{2}^{2}$ with $c \geqslant i+j+1$ and $u_{1}^{c} u_{2}^{3}$ with $c \geqslant i+j$.

Proof. We do this by downward induction on the filtration of the target term. There is nothing to prove if $i+1+j+2>m+n+3$ because both $u_{1}^{i} u_{2}^{j}$ and the target are zero. Assume we know this for all elements in higher filtration than $u_{1}^{i+1} u_{2}^{j+2}$. We know, from the previous lemma, that

$$
2\left(u_{1}^{i} u_{2}^{j}+u_{1}^{i-1} z u_{2}^{j-2}\right) \equiv u_{1}^{i+1} u_{2}^{j+2}
$$

modulo elements of higher filtration. By our induction we can take care of all of the elements of higher filtration except those listed modulo which we are working. We can only handle elements with the power of $u_{2}$ greater than or equal to 4 .

This lemma is one of our goals in this section and we get our other goal as an immediate corollary.

Corollary 15.6. If $n>m$, there is an element

$$
b_{1, n-1}=u_{1} u_{2}^{n-1}+u_{1}^{2} z \text { with } 2 b_{1, n-1}=0
$$

and the filtration of $u_{1}^{2} z$ is higher than that of $u_{1} u_{2}^{n-1}$.

Proof. From the lemma there is a $z^{\prime}$ of higher filtration than $u_{1} u_{2}^{n-1}$ such that $2\left(u_{1} u_{2}^{n-1}+z^{\prime}\right)=u_{1}^{2} u_{2}^{n+1}=0$. Since $2 u_{1} u_{2}^{n}=0$, we need not have any $u_{2}^{n}$ in any part of $z^{\prime}$. So, to have higher filtration than $u_{1} u_{2}^{n-1}$ we must have $u_{1}^{2}$ dividing $z^{\prime}$. 
Remark 15.7. A tactical mistake was made while trying to understand these computations. The "simple" test case that was studied at length was $R P^{16} \times R P^{16}$. This "easiest" case turned out to be significantly harder because $2 b_{1,7}=u_{1}^{8} u_{2}^{3}$. The shift to $m<n$ simplified things a lot.

\section{The spectral sequence for $\operatorname{ER}(2)^{*}\left(R P^{2 n} \wedge R P^{\infty}\right)$}

$E R(2)^{*}\left(R P^{2 n} \wedge R P^{\infty}\right)$ depends on $n$, but, as with $E R(2)^{*}\left(R P^{2 n}\right), d^{1}$ doesn't. Everything is still even degree so we only have to worry about the 4 odd differentials.

Theorem 16.1. In the Bockstein spectral sequence for $E R(2)^{*}\left(R P^{2 n} \wedge R P^{\infty}\right)$ where $3<n$ we have (with differentials all modulo higher filtrations):

$E^{1}$ is

$$
v_{2}^{s} \alpha^{k} u_{1}^{i} u_{2}, \quad 0 \leqslant s<8, \quad 0 \leqslant k, \quad 0<i \leqslant n
$$

and

$$
\begin{gathered}
v_{2}^{s} u_{1}^{i} u_{2}^{j}, \quad 0 \leqslant s<8, \quad 0<i \leqslant n, \quad 1<j ; \\
d^{1}\left(v_{2}^{2 s-5} \alpha^{k} u_{1}^{i} u_{2}\right) \equiv v_{2}^{2 s} \alpha^{k+1} u_{1}^{i+1} u_{2} \text { for } 0 \leqslant i<n ; \\
d^{1}\left(v_{2}^{2 s-5} u_{1}^{i} u_{2}^{j}\right) \equiv v_{2}^{2 s} u_{1}^{i+1} u_{2}^{j+2} ;
\end{gathered}
$$

for $0<i<n$ and $1<j$.

$E^{2}=E^{3}$ is:

$$
\begin{gathered}
v_{2}^{2 s} \alpha^{k} u_{1} u_{2}, \quad k \geqslant 0 ; \\
v_{2}^{2 s} u_{1}^{i} u_{2}^{\{1,2,3\}}, \quad 1<i \leqslant n ; \\
v_{2}^{2 s} u_{1} u_{2}^{j}, \quad 1<j ; \\
v_{2}^{2 s+1} \alpha^{k} u_{1}^{n} u_{2} ; \\
v_{2}^{2 s+1} u_{1}^{n} u_{2}^{j}, \quad 1<j ; \\
d^{3}\left(v_{2}^{\{2,6\}} \alpha^{k} u_{1} u_{2}\right)=v_{2}^{\{4,0\}} \alpha^{k+1} u_{1} u_{2} ; \\
d^{3}\left(v_{2}^{\{2,6\}} u_{1}^{i} u_{2}^{\{1,2,3\}}\right) \equiv v_{2}^{\{4,0\}} u_{1}^{i+2} u_{2}^{\{1,2,3\}}, \quad 1<i<n-1 ; \\
d^{3}\left(v_{2}^{\{2,6\}} u_{1} u_{2}^{j}\right) \equiv v_{2}^{\{4,0\}} u_{1} u_{2}^{j+2} .
\end{gathered}
$$

For $n \equiv 1,2,5$ or $6 \bmod 8$ :

$$
\begin{gathered}
d^{3}\left(v_{2}^{\{3,7\}} \alpha^{k} u_{1}^{n} u_{2}\right)=v_{2}^{\{5,1\}} \alpha^{k+1} u_{1}^{n} u_{2} ; \\
d^{3}\left(v_{2}^{\{3,7\}} u_{1}^{n} u_{2}^{j}\right) \equiv v_{2}^{\{5,1\}} u_{1}^{n} u_{2}^{j+2}, \quad 1<j .
\end{gathered}
$$

For $n \equiv 3,4,7$ or $8 \bmod 8$ :

$$
\begin{gathered}
d^{3}\left(v_{2}^{\{1,5\}} \alpha^{k} u_{1}^{n} u_{2}\right)=v_{2}^{\{3,7\}} \alpha^{k+1} u_{1}^{n} u_{2} ; \\
d^{3}\left(v_{2}^{\{1,5\}} u_{1}^{n} u_{2}^{j}\right) \equiv v_{2}^{\{3,7\}} u_{1}^{n} u_{2}^{j+2}, \quad 1<j .
\end{gathered}
$$




$$
E^{4}=E^{5} \text { is: }
$$

$$
\begin{gathered}
v_{2}^{\{0,4\}} u_{1}^{i} u_{2}^{j}, \quad 0<i<4, \quad 0<j<4 ; \\
v_{2}^{\{2,6\}} u_{1}^{\{n-1, n\}} u_{2}^{\{1,2,3\}} .
\end{gathered}
$$

For $n \equiv 1,2,5$ or $6 \bmod 8$ :

$$
v_{2}^{\{5,1\}} u_{1}^{n} u_{2}^{\{1,2,3\}}
$$

For $n \equiv 3,4,7$ or $8 \bmod 8$ :

$$
v_{2}^{\{3,7\}} u_{1}^{n} u_{2}^{\{1,2,3\}}
$$

For $n \equiv 1,2,5$ or $6 \bmod 8$ :

$$
d^{5}\left(v_{2}^{\{2,6\}} u_{1}^{n-1} u_{2}^{\{1,2,3\}}\right)=v_{2}^{\{1,5\}} u_{1}^{n} u_{2}^{\{1,2,3\}} .
$$

For $n \equiv 1,2,5$ or $6 \bmod 8$ :

$$
E^{6}=E^{7} \text { is: }
$$

$$
\begin{gathered}
v_{2}^{\{0,4\}} u_{1}^{i} u_{2}^{j}, \quad 0<i<4, \quad 0<j<4 \\
v_{2}^{\{2,6\}} u_{1}^{n} u_{2}^{\{1,2,3\}} \\
d^{7}\left(v_{2}^{4} u_{1}^{i} u_{2}^{j}\right)=u_{1}^{i} u_{2}^{j} .
\end{gathered}
$$

For $n \equiv 1$ or 6 modulo 8 :

$$
d^{7}\left(v_{2}^{2} u_{1}^{n} u_{2}^{\{1,2,3\}}\right)=v_{2}^{6} u_{1}^{n} u_{2}^{\{1,2,3\}},
$$

and for $n \equiv 2$ or 5 modulo 8 :

$$
d^{7}\left(v_{2}^{6} u_{1}^{n} u_{2}^{\{1,2,3\}}\right)=v_{2}^{2} u_{1}^{n} u_{2}^{\{1,2,3\}} .
$$

For $n \equiv 3,4,7$ or $8 \bmod 8$ :

$$
E^{5}=E^{6}=E^{7} \text {; }
$$

$$
d^{7}\left(v_{2}^{4} u_{1}^{i} u_{2}^{j}\right)=u_{1}^{i} u_{2}^{j}
$$

For $n \equiv 3$ or $4 \bmod 8$

$$
\begin{aligned}
d^{7}\left(v_{2}^{6} u_{1}^{\{n-1, n\}} u_{2}^{\{1,2,3\}}\right) & =v_{2}^{2} u_{1}^{\{n-1, n\}} u_{2}^{\{1,2,3\}} ; \\
d^{7}\left(v_{2}^{3} u_{1}^{n} u_{2}^{\{1,2,3\}}\right) & =v_{2}^{7} u^{n} u_{2}^{\{1,2,3\}} .
\end{aligned}
$$

For $n \equiv 7$ or $8 \bmod 8$

$$
\begin{aligned}
d^{7}\left(v_{2}^{2} u_{1}^{\{n-1, n\}} u_{2}^{\{1,2,3\}}\right) & =v_{2}^{6} u_{1}^{\{n-1, n\}} u_{2}^{\{1,2,3\}} ; \\
d^{7}\left(v_{2}^{7} u_{1}^{n} u_{2}^{\{1,2,3\}}\right) & =v_{2}^{3} u_{1}^{n} u_{2}^{\{1,2,3\}} .
\end{aligned}
$$

Proof. The computation of $d^{1}$ is made possible by Lemma 15.5. The higher differentials all come from products where the differential on $R P^{2 n}$ is the one used.

Corollary 16.2. Let $m=8 K$ and $3<n$. In the Bockstein spectral sequence for $E R(2)^{*}\left(R P^{2 n} \wedge R P^{\infty} / R P^{2 m}\right)$ we have the same result as above; just multiply everything by $u_{2}^{m}$. 


\section{The Bockstein spectral sequence for $\operatorname{ER}(2)^{*}\left(R P^{\infty} \wedge R P^{\infty}\right)$}

We know from Theorem 3.4 that

$$
E R(2)^{*}\left(R P^{\infty} \wedge R P^{\infty}\right) \simeq E R(2)^{*}\left(R P^{\infty}\right) \hat{\otimes}_{E R(2)^{*}} E R(2)^{*}\left(R P^{\infty}\right)
$$

We can write down the entire Bockstein spectral sequence for this as a Corollary to the previous section just by letting $n$ go off to infinity. We also want to see the elements which represent things in our spectral sequence.

Theorem 17.1. In the Bockstein spectral sequence for $E R(2)^{*}\left(R P^{\infty} \wedge R P^{\infty}\right)$ we have, where everything is modulo higher filtrations:

$E^{1}$ is

$$
\begin{gathered}
v_{2}^{s} \alpha^{k} u_{1}^{i} u_{2}, \quad 0 \leqslant s<8, \quad 0 \leqslant k, \quad 0<i ; \\
v_{2}^{s} u_{1}^{i} u_{2}^{j}, \quad 0 \leqslant s<8, \quad 0<i, \quad 1<j ; \\
d^{1}\left(v_{2}^{2 s-5} \alpha^{k} u_{1}^{i} u_{2}\right)=2 v_{2}^{2 s} \alpha^{k} u_{1}^{i} u_{2} \equiv v_{2}^{2 s} \alpha^{k+1} u_{1}^{i+1} u_{2} ; \\
d^{1}\left(v_{2}^{2 s-5} u_{1}^{i} u_{2}^{j}\right)=2 v_{2}^{2 s} u_{1}^{i} u_{2}^{j} \equiv v_{2}^{2 s} u_{1}^{i+1} u_{2}^{j+2}, \quad 0<i, \quad 1<j . \\
E^{2}=E^{3} i s \quad v_{2}^{2 s} u_{1}^{i} u_{2}^{\{1,2,3\}}, \quad 1<i ; \quad v_{2}^{2 s} u_{1} u_{2}^{j}, \quad 1<j ; \\
v_{2}^{2 s} \alpha^{k} u_{1} u_{2}, \quad 0 \leqslant k ; \quad{ }^{3}\left(v_{2}^{\{2,6\}} \alpha^{k} u_{1} u_{2}\right)=v_{2}^{\{4,0\}} \alpha^{k+1} u_{1} u_{2} ; \\
d^{3}\left(v_{2}^{\{2,6\}} u_{1}^{i} u_{2}^{\{1,2,3\}}\right) \equiv v_{2}^{\{4,0\}} u_{1}^{i+2} u_{2}^{\{1,2,3\}}, \quad 1<i ; \\
d^{3}\left(v_{2}^{\{2,6\}} u_{1} u_{2}^{j}\right) \equiv v_{2}^{\{4,0\}} u_{1} u_{2}^{j+2}, \quad 1<j . \\
E^{4}=E^{5}=E^{6}=E^{7} \quad v_{2}^{\{0,4\}} u_{1}^{i} u_{2}^{j}, \quad 0<i<4, \quad 0<j<4 ; \\
d^{7}\left(v_{2}^{4} u_{1}^{i} u_{2}^{j}\right)=u_{1}^{i} u_{2}^{j} .
\end{gathered}
$$

The $x^{1}$-torsion is given by

$$
\begin{gathered}
\alpha_{s} \alpha^{k} u_{1}^{i} u_{2} \longrightarrow 2 v_{2}^{2 s} \alpha^{k} u_{1}^{i} u_{2} \equiv v_{2}^{2 s} \alpha^{k+1} u_{1}^{i+1} u_{2} \\
\alpha_{s} u_{1}^{i} u_{2}^{j} \longrightarrow 2 v_{2}^{2 s} u_{1}^{i} u_{2}^{j} \equiv v_{2}^{2 s} u_{1}^{i+1} u_{2}^{j+2}, \quad 0<i, \quad 1<j .
\end{gathered}
$$

The $x^{3}$-torsion is given by

$$
\begin{gathered}
\alpha^{k} u_{1} u_{2} \longrightarrow \alpha^{k} u_{1} u_{2}, \quad 0<k ; \\
w \alpha^{k} u_{1} u_{2} \longrightarrow v_{2}^{4} \alpha^{k+1} u_{1} u_{2}, \quad 0 \leqslant k ; \\
u_{1}^{i+2} u_{2}^{\{1,2,3\}} \longrightarrow u_{1}^{i+2} u_{2}^{\{1,2,3\}}, \quad 1<i ; \\
w u_{1}^{i} u_{2}^{\{1,2,3\}} \longrightarrow v_{2}^{4} u_{1}^{i+2} u_{2}, \quad 1<i ; \\
u_{1} u_{2}^{j} \longrightarrow u_{1} u_{2}^{j}, \quad 3<j ; \\
w u_{1} u_{2}^{j} \longrightarrow v_{2}^{4} u_{1} u_{2}^{j+2}, \quad 1<j .
\end{gathered}
$$

The $x^{7}$-torsion is given by

$$
u_{1}^{i} u_{2}^{j} \longrightarrow u_{1}^{i} u_{2}^{j}, \quad i<4, \quad j<4 .
$$


Proof. The differentials follow from the previous section. The elements described in $E R(2)^{*}\left(R P^{\infty} \wedge R P^{\infty}\right)$ have the appropriate torsion and map to the correct elements in $E(2)^{*}\left(R P^{\infty} \wedge R P^{\infty}\right)$.

Remark 17.2. Note that we have no elements divisible by $w u_{1}^{2} u_{2}^{4}$. The element $u_{2}^{4}$ can be replaced using (1.3) and this can be rewritten in terms of other elements.

Corollary 17.3. The map $E R(2)^{*}(-) \rightarrow E(2)^{*}(-)$ induces an isomorphism

$$
E R(2)^{16 *}\left(R P^{\infty} \wedge R P^{\infty}\right) \longrightarrow E(2)^{16 *}\left(R P^{\infty} \wedge R P^{\infty}\right)
$$

Proof. $E(2)^{16 *}\left(R P^{\infty} \wedge R P^{\infty}\right)$ has, for a 2-adic basis, $\alpha^{i} u_{1}^{i} u_{2}$ and $u_{1}^{i} u_{2}^{j}$ for $j>1$. Since $\alpha, u_{1}$ and $u_{2}$ all come from $E R(2)^{*}\left(R P^{\infty} \wedge R P^{\infty}\right)$, we have a surjection. From the Bockstein spectral sequence for $E R(2)^{*}\left(R P^{\infty} \wedge R P^{\infty}\right)$ we can just read off all of the elements in degree 16*. From the $x^{1}$-torsion we have, modulo higher filtrations,

$$
\begin{gathered}
\alpha_{0} \alpha^{k} u_{1}^{i} u_{2} \equiv \alpha^{k+1} u_{1}^{i+1} u_{2} ; \\
\alpha_{0} u_{1}^{i} u_{2}^{j} \equiv u_{1}^{i+1} u_{2}^{j+2}, \quad 0<i, \quad 1<j .
\end{gathered}
$$

From the $x^{3}$-torsion we have

$$
\alpha^{k} u_{1} u_{2}, \quad 0<k ; \quad u_{1}^{i+2} u_{2}^{\{1,2,3\}}, \quad 1<i ; \quad u_{1} u_{2}^{j}, \quad 3<j .
$$

Finally, from the $x^{7}$-torsion we have

$$
u_{1}^{i} u_{2}^{j}, \quad i<4, \quad j<4
$$

Combining all of these elements we get exactly what we need. In particular, there are no elements divisible by $x$ in degrees $16 *$.

Remark 17.4. In the next paper, we will need the slightly more delicate fact that $E R(2)^{16 *+8}\left(R P^{\infty} \wedge R P^{\infty}\right)$ injects into $E(2)^{16 *+8}\left(R P^{\infty} \wedge R P^{\infty}\right)$.

\section{A special element}

To extract the information we need from the Bockstein spectral sequence for $E R(2)^{*}\left(R P^{2 n} \wedge R P^{2 m}\right)$ we need to deal with odd degree elements for the first time. Our approach to this will be to use the long exact sequence coming from:

$$
R P^{2 n} \wedge R P^{2 m} \longrightarrow R P^{2 n} \wedge R P^{\infty} \longrightarrow R P^{2 n} \wedge R P^{\infty} / R P^{2 m} \text {. }
$$

From Section 16 we know $E R(2)^{*}(-)$ for the two terms on the right (when $2 m=$ $16 K)$ and we will compute a special element in the kernel. Many thanks to Jesus González for his work with the second author on $B P^{*}\left(R P^{2 n} \wedge R P^{2 m}\right)$. Ideas from there translated nicely to this situation and saved us from many a contorted filtration.

Recall

$$
[2](u)=\sum_{k \geqslant 0} a_{k} u^{k+1}
$$

in degree -16 .

Definition 18.1. Let $\epsilon(n)$ be 0 for $n \equiv 7$ or $0 \bmod 8,1$ for $n \equiv 1$ or $6 \bmod 8,2$ for $n \equiv 2$ or $5 \bmod 8$, and 3 for $n \equiv 3$ or $4 \bmod 8$. These are just the numbers such that $0 \neq u^{n+\epsilon(n)} \in E R(2)^{*}\left(R P^{2 n}\right)$ and $0=u^{n+\epsilon(n)+1}$. 
Theorem 18.2. Let $m=8 K$, and $m<n$. Define the degree $-16(n+1)$ element

$$
g_{0}=\sum_{i=0}^{m-1} u_{1}^{n-m+1+i} \sum_{k=i+1}^{\infty} a_{k} u_{2}^{m-i+k} .
$$

The element $u_{1}^{\epsilon(n)} g_{0}$ is in the kernel of the map

$$
E R(2)^{*}\left(R P^{2 n} \wedge R P^{\infty} / R P^{2 m}\right) \longrightarrow E R(2)^{*}\left(R P^{2 n} \wedge R P^{\infty}\right) .
$$

The elements $u_{1}^{i} g_{0}, 0 \leqslant i<m$, are nonzero, not divisible by $x$ and $x^{2} u_{1}^{i} g_{0} \neq 0$. For $n \equiv 1,2,5$ and $6 \bmod 8, \alpha^{k} u_{1}^{m} g_{0} \neq 0$. For $n \equiv 2,3,4$ and $5 \bmod 8, u_{1}^{m-1+\epsilon(n)} g_{0} \neq 0$.

Proof. The map to $E R(2)^{*}\left(R P^{2 n} \wedge R P^{\infty}\right)$ takes $g_{0}$ to an element with the same notation. To see that $u_{1}^{\epsilon(n)} g_{0}$ is in the kernel we will add 0 to it in the form of $u_{1}^{\epsilon(n)}$ times

$$
g_{1}=\sum_{i=0}^{m-1} u_{1}^{n-m+1+i} \sum_{k=0}^{i} a_{k} u_{2}^{m-i+k} .
$$

Fix $q=m-i+k, 0<q \leqslant m$. Then $i=m-q+k$ and we look at the coefficient of $u_{2}^{q}$ in $u_{1}^{\epsilon(n)} g_{1}$ :

$$
\sum_{k=0}^{m-1} a_{k} u_{1}^{n+1-q+k+\epsilon(n)}
$$

This is zero because it is the relation in $E R(2)^{*}\left(R P^{2 n}\right)$. Adding, we have

$$
g_{0}+g_{1}=\sum_{i=0}^{m-1} u_{1}^{n-m+1+i} \sum_{k=0}^{\infty} a_{k} u_{2}^{m-i+k}
$$

where the sum

$$
\sum_{k=0}^{\infty} a_{k} u_{2}^{m-i+k}=0
$$

This shows that $u_{1}^{\epsilon(n)} g_{0}$ is in the kernel. Although the image of $g_{0}$, when added to $g_{1}$ is zero, $g_{1}$ isn't zero until it has been multiplied by $u_{1}^{\epsilon(n)}$ so $g_{0}$ is not in the kernel until it too has been multiplied by $u_{1}^{\epsilon(n)}$.

Multiply $g_{0}$ by $u_{1}^{m-1}$ to get

$$
u_{1}^{m-1} g_{0}=\sum_{i=0}^{m-1} u_{1}^{n+i} \sum_{k=i+1}^{\infty} a_{k} u_{2}^{m-i+k} .
$$

Since $u_{1}^{n+1}$ is divisible by $x$, if we reduce modulo $x$ all we have left is:

$$
u_{1}^{m-1} g_{0} \equiv u_{1}^{n} \sum_{k=1}^{\infty} a_{k} u_{2}^{m+k} .
$$

We need to show that this element is not divisible by $x$ and that $x^{2}$ times it is nonzero. We use the algorithm in Remark 15.3. The first term in the sum, $a_{1} u_{1}^{n} u_{2}^{m+1}=$ $\alpha u_{1}^{n} u_{2}^{m+1}$, represents an $x^{1}$-torsion generator in the spectral sequence of Corol- 
lary 16.2. The element $a_{3} u_{1}^{n} u_{2}^{m+3}$ is an $x^{3}$-torsion element. Any $a_{k}$ divisible by 2 , such as $a_{2}$, has that 2 applied to $u_{1}^{n}$ and the element becomes divisible by $x$. All we have left to consider are elements $a_{k}$ that are powers of $\alpha$. In this case we know that $k>3$. Since we can work mod 2 , the algorithm just uses $\alpha u_{2}^{2} \equiv u_{2}^{4}$ modulo higher powers of $u_{2}$. All such elements end up as $u_{1}^{n} u_{2}^{j}$ with $j>3$ and as such are $x^{1}$-torsion elements. We can conclude that our element is not divisible by $x$ and that $x^{2}$ times it is nonzero.

Next we deal with the $n \equiv 3$ and $4 \bmod 8$ cases when we know $u_{1}^{n+3} \neq 0$. Multiply $g_{0}$ by $u_{1}^{m+2}$ to get

$$
u_{1}^{m+2} g_{0}=u_{1}^{n+3} \sum_{k=1}^{\infty} a_{k} u_{2}^{m+k}
$$

We know that $u_{1}^{n+3}$ is divisible by $x^{6}$ so both 2 and $\alpha$ times it are zero. The only $a_{k}$ without a 2 or an $\alpha$ is $a_{3}$ so this reduces to $u_{1}^{n+3} u_{2}^{m+3}$. This is represented by $x^{6} v_{2}^{7} u_{1}^{n} u_{2}^{m+3}$ in the spectral sequence and is nonzero.

For all other $n, u_{1}^{n+3}$ is zero.

Let $n \equiv 2$ or $5 \bmod 8$. We know that $u_{1}^{n+2}$ is nonzero and is $x^{4}$ times the element in the Bockstein spectral sequence for $E R(2)^{*}\left(R P^{2 n}\right)$ represented by $v_{2}^{2} u_{1}^{n}$. We also know that $u_{1}^{n+3}=0$. Now multiply $g_{0}$ by $u_{1}^{m+1}$ to get

$$
u_{1}^{m+1} g_{0}=u_{1}^{n+2} \sum_{k=1}^{\infty} a_{k} u_{2}^{m+k} .
$$

Since our $u_{1}^{n+2} \in E R(2)^{*}\left(R P^{2 n}\right)$ is divisible by $x^{4}$, both 2 and $\alpha$ times $u_{1}^{n+2}$ give zero. Recall also that every $a_{k}$ has a 2 or $\alpha$ in it except for $a_{3}$. Our formula is now just:

$$
u_{1}^{m+1} g_{0}=u_{1}^{n+2} u_{2}^{m+3}
$$

In the Bockstein spectral sequence for $E R(2)^{*}\left(R P^{2 n} \wedge R P^{\infty} / R P^{2 m}\right)$ the element representing $u_{1}^{n+2} u_{2}^{m+3}$ is $x^{4}$ times $v_{2}^{2} u_{1}^{n} u_{2}^{m+3}$ which is the target of a $d^{7}$ so this is nonzero.

We want to do a bit more for $u_{1}^{m} g_{0}$ because we want $\alpha^{j} u_{1}^{m} g_{0}$ when $n \equiv 1,2,5$ and $6 \bmod 8$. We know that $u_{1}^{n+2}$ is divisible by $x^{4}$ if it is nonzero so $\alpha$ will kill it. So, for $j>0, \alpha^{j} u_{1}^{m} g_{0}$ is:

$$
\alpha^{j} u_{1}^{n+1} \sum_{k=1}^{\infty} a_{k} u_{2}^{m+k}
$$

Any 2 in $a_{k}$ will raise the power of $u_{1}$ and give us $x^{4}$ killing the $\alpha$, so, as in the previous cases, we are left with $\alpha u_{2}^{m+1}+u_{2}^{m+3}$ and higher powers of $u_{2}$. Since we have an $\alpha$, the $u_{2}^{m+3}$ also goes away and we are left with $\alpha^{j+1} u_{1}^{n+1} u_{2}^{m+1}$. These elements are represented by $x^{2} v_{2}^{5} \alpha^{j+1} u_{1}^{n} u_{2}^{m+1}$ in the spectral sequence and are all nonzero. 


\section{Starting the spectral sequence for $\operatorname{ER}(2)^{*}\left(R P^{2 n} \wedge R P^{2 m}\right)$}

In the previous section we found a special element

$$
g_{0} \in E R(2)^{-16(n+1)}\left(R P^{2 n} \wedge R P^{\infty} / R P^{2 m}\right),
$$

where $m=8 K$, such that $u_{1}^{\epsilon(n)} g_{0}$ went to zero in $E R(2)^{*}\left(R P^{2 n} \wedge R P^{\infty}\right)$. From the long exact sequence for the cofibration,

$$
R P^{2 n} \wedge R P^{2 m} \longrightarrow R P^{2 n} \wedge R P^{\infty} \longrightarrow R P^{2 n} \wedge R P^{\infty} / R P^{2 m},
$$

we must have an element $\hat{g}_{0}(n) \in E R(2)^{-16(n+\epsilon(n))-17}\left(R P^{2 n} \wedge R P^{2 m}\right)$ such that $\partial\left(\hat{g}_{0}(n)\right)=u_{1}^{\epsilon(n)} g_{0}$. Because $u_{1}^{i} g_{0}$ is not divisible by $x$ for $0 \leqslant i<m$, the same must be true of $u_{1}^{i-\epsilon(n)} \hat{g}_{0}(n)$ and that means these elements must reduce non-trivially to $E(2)^{*}\left(R P^{2 n} \wedge R P^{2 m}\right)$. The only elements in degree $-1 \bmod 16$ are $\alpha^{k} u_{1}^{i} z_{-16 n-17}$, with $0 \leqslant i<m$. The only elements in exactly degree $-16(n+\epsilon(n))-17$ with these

$u_{1}^{i-\epsilon(n)}$ nonzero are $\alpha^{3 k} u_{1}^{\epsilon(n)} z_{-16 n-17}$ and so $\hat{g}_{0}(n)$ must reduce to some combination of these elements.

Theorem 19.2. The Bockstein spectral sequence for $E R(2)^{*}\left(R P^{2 m} \wedge R P^{2 n}\right)$ when $m \leqslant 8 K<n$ :

$$
E^{1}:
$$

$E(2)^{*}\left(R P^{2 m} \wedge R P^{2 n}\right) \simeq E(2)^{*}\left(R P^{2 m}\right) \otimes_{E(2)^{*}} E(2)^{*}\left(R P^{2 n}\right) \oplus \Sigma^{-16 n-1} E(2)^{*}\left(R P^{2 m}\right)$

has a 2-adic basis

$$
\begin{gathered}
v_{2}^{s} \alpha^{k} u_{1}^{i} u_{2}, \quad 0 \leqslant k, \quad 0<i \leqslant m, \quad s<8 \\
v_{2}^{s} u_{1}^{i} u_{2}^{j}, \quad 0<i \leqslant m, \quad 1<j \leqslant n, \quad s<8
\end{gathered}
$$

and

$$
v_{2}^{s} \alpha^{k} u_{1}^{i} z_{-16 n-17}, \quad 0 \leqslant k, \quad 0 \leqslant i<m, \quad s<8 .
$$

There is an element

$$
\hat{g}_{0}(n) \in E R(2)^{-16(n+\epsilon(n))-17}\left(R P^{2 m} \wedge R P^{2 n}\right) \simeq E R(2)^{-16(n+\epsilon(n))-17}\left(R P^{2 n} \wedge R P^{2 m}\right)
$$

with

$$
0 \neq \partial\left(\hat{g}_{0}(n)\right) \in E R(2)^{-16(n+\epsilon(n))-16}\left(R P^{2 n} \wedge R P^{\infty} / R P^{2 m}\right)
$$

such that $\hat{g}_{0}(n)$ reduces to

$$
u_{1}^{\epsilon(n)} z_{-16 n-17} \in E(2)^{-16 n-17}\left(R P^{2 m} \wedge R P^{2 n}\right)
$$

and the $\hat{g}_{0}(n)$ are compatible with the maps $R P^{2(m-1)} \rightarrow R P^{2 m}$.

Modulo terms of higher filtration, $d^{1}$ is

$$
\begin{gathered}
d^{1}\left(v_{2}^{2 s-5} \alpha^{k} u_{1}^{i} u_{2}\right) \equiv v_{2}^{2 s} \alpha^{k+1} u_{1}^{i+1} u_{2}, \quad 0<i<m ; \\
d^{1}\left(v_{2}^{2 s-5} u_{1}^{i} u_{2}^{j}\right) \equiv v_{2}^{2 s} u_{1}^{i+1} u_{2}^{j+2}, \quad 0<i<m, \quad 1<j<n-1 ; \\
d^{1}\left(v_{2}^{2 s-5} \alpha^{k} u_{1}^{i} z_{-16 n-17}\right) \equiv v_{2}^{2 s} \alpha^{k+1} u_{1}^{i+1} z_{-16 n-17}, \quad 0 \leqslant i<m-1 .
\end{gathered}
$$


$E^{2}$ is:

$$
\begin{array}{cc}
v_{2}^{2 s} \alpha^{k} u_{1} u_{2}, & 0 \leqslant k ; \\
v_{2}^{2 s} u_{1}^{i} u_{2}^{\{1,2,3\}}, & 1<i<m ; \\
v_{2}^{2 s} u_{1} u_{2}^{j}, & 1<j \leqslant n ; \\
v_{2}^{2 s+1} \alpha^{k} u_{1}^{m} u_{2}, & 0<k ; \\
v_{2}^{2 s+1} u_{1}^{m} u_{2}^{j}, & 0<j \leqslant n ; \\
v_{2}^{2 s+1} u_{1}^{i} u_{2}^{n}, & 0<i<m ; \\
v_{2}^{2 s+1} u_{1}^{i} b_{1, n-1}, & 0 \leqslant i<m-1 ; \\
v_{2}^{2 s} \alpha^{k} z_{-16 n-17} ; & \\
v_{2}^{2 s} u_{1}^{i} z_{-16 n-17}, & 0<i<m ; \\
v_{2}^{2 s+1} \alpha^{k} u_{1}^{m-1} z_{-16 n-17 .} &
\end{array}
$$

Proof. There are a couple of things to prove here. We must evaluate $d^{1}$ and get $E^{2}$ and then we must verify the reduction of $\hat{g}_{0}(n)$ and prove its naturality.

The differential, $d^{1}$, is even degree so it acts independently on the even and odd degree parts of $E^{1}$. On the even degree part, $d^{1}$ is induced from $R P^{\infty} \wedge R P^{\infty}$. It is only the third line, the differential on the odd degree elements, that we need to prove. If we can show that $d^{1}\left(z_{-16 n-17}\right)=0$, then the differential will follow from its behavior on the coefficients $v_{2}^{s}$.

The cofibration (19.1) gives a long exact sequence in $E(2)^{*}(-)$. The two terms with $R P^{\infty}$ and $R P^{\infty} / R P^{2 m}$ are in even degrees so all of the even degree elements of $E(2)^{*}\left(R P^{2 n} \wedge R P^{2 m}\right)$ come from $E(2)^{*}\left(R P^{2 n} \wedge R P^{\infty}\right)$ and all of the odd degree elements have boundary non-trivial and inject into $E(2)^{*}\left(R P^{2 n} \wedge R P^{\infty} / R P^{2 m}\right)$. The boundary is induced by the map

$$
R P^{2 n} \wedge R P^{\infty} / R P^{2 m} \longrightarrow R P^{2 n} \wedge \Sigma R P^{2 m}
$$

The image of $z_{-16 n-17}$ is in degree $0 \bmod 16$ and so its representation must have a $v_{2}^{0}$. All of $d^{1}$ for $R P^{2 n} \wedge R P^{\infty} / R P^{2 m}$ is on odd powers of $v_{2}$ so since we have the odd degree elements injecting and $d^{1}$ on the image of $z_{-16 n-17}$ equal to zero, we must have $d^{1}\left(z_{-16 n-17}\right)=0$. The result for $d^{1}$ follows as described above.

We already know the reduction of $\hat{g}_{0}(n)$ is of the form $\alpha^{3 k} u_{1}^{\epsilon(n)} z_{-16 n-17}$ so all we need to do is show that $k=0$. Since we have computed $d^{1}$ already, we know that $\alpha^{3 k} u_{1}^{m-1} z_{-16 n-17}$ is in the image of $d^{1}$ for $k>0$. The class $\alpha^{3 k} u_{1}^{m-1} z_{-16 n-17}$ represents the element $u_{1}^{m-1-\epsilon(n)} \hat{g}_{0}(n)$ and we know this has $x^{2}$ on it nonzero. We just showed that all of these elements with $k>0$ are $x^{1}$-torsion so we must have $k=0$ and $\hat{g}_{0}(n)$ maps to $u_{1}^{\epsilon(n)} z_{-16 n-17}$, with, if necessary, a little redefinition of $z_{-16 n-17}$ to avoid a sum.

Consider the diagram:

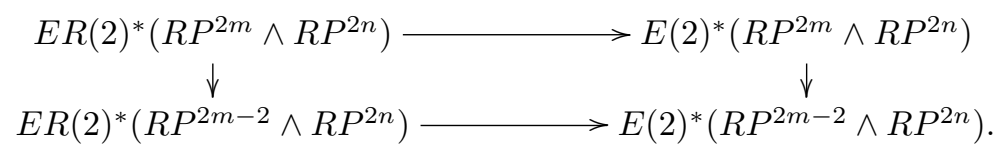


By naturality, Remark 14.2, in $E(2)^{*}(-), z_{-16 n-17}$ maps to the element of the same name on the right hand map. The element $\hat{g}_{0}(n)$ in the upper left corner must factor through a $\hat{g}_{0}(n)$ in the lower left corner. It isn't obvious that $\partial\left(\hat{g}_{0}(n)\right)$ must be nonzero though. If it were zero, then $\hat{g}_{0}(n)$ would have to come from

$$
E R(2)^{-16(n+\epsilon(n))-17}\left(R P^{2 n} \wedge R P^{\infty}\right) .
$$

We know that in here any odd degree elements are divisible by $x$ but we also know that $\hat{g}_{0}(n)$ is not divisible by $x$ because it reduces to $z_{-16 n-17}$.

It is an instructive exercise to apply the algorithm to see how the element $g_{0}$ behaves under the map induced by $R P^{8 K-8} \rightarrow R P^{8 K}$.

Our goal with products all along has been to prove:

Proposition 19.3. When $n \equiv 1,2,5$ or 6 modulo $8, m \leqslant 8 K$, and $8 K+8<n$, the element $u_{1}^{m} u_{2}^{n+1} \in E R(2)^{*}\left(R P^{2 m} \wedge R P^{2 n}\right)$ is nonzero.

Proof. $u_{2}^{n+1} \in E R(2)^{*}\left(R P^{2 n}\right)$ is represented by $x^{2}$ times the element represented in the spectral sequence by $v_{2}^{5} u_{2}^{n}$. So $u_{1}^{m} u_{2}^{n+1}$ is $x^{2} z$ where $z$ reduces to $u_{1}^{m} u_{2}^{n} v_{2}^{5}$ in the Bockstein spectral sequence. The element $u_{1}^{m} u_{2}^{n} v_{2}^{5}$ survives to $E^{2}$. For $z$ to have $x^{2} z \neq 0$ it is enough that $u_{1}^{m} u_{2}^{n} v_{2}^{5}$ survives to $E^{3}$, i.e. that it is not hit by a $d^{2}$. (It cannot be the source of any differential because it is the product of the elements represented by $u_{1}^{m}$ and $u_{2}^{n} v_{2}^{5}$.)

The differential $d^{2}$ has degree $35 \equiv-13$. Our element $u_{1}^{m} u_{2}^{n} v_{2}^{5}$ has degree $-16(m+$ $n)-30$ so the source that would have to hit it would have to have degree $-16(m+$ $n)-17$; in particular, it must be odd degree. The odd degree elements in the $E^{2}$-term of our Bockstein spectral sequence are

$$
\begin{gathered}
v_{2}^{2 s} \alpha^{k} z_{-16 n-17} \\
v_{2}^{2 s} u_{1}^{i} z_{-16 n-17 \quad 0<i<m ;} \\
\text { and } \quad v_{2}^{2 s+1} \alpha^{k} u_{1}^{m-1} z_{-16 n-17} .
\end{gathered}
$$

The only elements with degree equal to -1 modulo 16 are

$$
\begin{gathered}
\alpha^{k} z_{-16 n-17} ; \\
\text { and } \quad u_{1}^{i} z_{-16 n-17}, \quad 0<i<m .
\end{gathered}
$$

Since our differentials commute with multiplication by $\alpha$ and $u_{1}$, if such a differential exists it has to be non-trivial on $z_{-16 n-17}$. Because $u_{1}^{\epsilon(n)} z_{-16 n-17}$ is in the image of $\hat{g}_{0}(n)$, it must have all differentials on it trivial. Thus the target, $d^{2}\left(z_{-16 n-17}\right)$ must be killed by $u_{1}^{\epsilon(n)}$, i.e. $u_{1}^{\epsilon(n)} d^{2}\left(z_{-16 n-17}\right)=d^{2}\left(u_{1}^{\epsilon(n)} z_{-16 n-17}\right)=0$. If we do have a non-trivial $d^{2}$ differential on our $z_{-16 n-17}$ for $m=8 K+8$, we can read off all the possible targets from Theorem 19.2, keeping in mind what the degree must be and that $u_{1}^{\epsilon(n)}$ must kill it. This requires an odd power of $v_{2}$ and a high power of $u_{1}$. Because of the high power of $u_{1}$, all such possible targets will, by naturality of the spectral sequence, Remark 14.2, go to zero in $m=8 K$ and so our differential must be trivial there. 
Corollary 19.4. In $E R(2)^{*}\left(R P^{2 m} \wedge R P^{2 n}\right), m \leqslant 8 K, 8 K+8<n, n \equiv 1,2,5$ and 6 modulo 8, the following elements are nonzero and independent in our 2-adic representation:

$$
\alpha^{k} u_{1}^{i} u_{2}, \quad k \geqslant 0, \quad i \leqslant m
$$

and

$$
u_{1}^{i} u_{2}^{j}, \quad i \leqslant m, \quad j \leqslant n+1 .
$$

Furthermore, $u_{1}^{i} u_{2}^{n+2}=0$ when $i \geqslant 4, n>8 K+8$.

Proof. The elements $\alpha^{k} u_{1}^{i} u_{2}, u_{1}^{i} u_{2}^{j}, i \leqslant m, j \leqslant n$, reduce to $E(2)^{*}\left(R P^{2 m} \wedge R P^{2 n}\right)$. All we have left to worry about are the elements $u_{1}^{i} u_{2}^{n+1}, i \leqslant m$. We know $u_{2}^{n+1}=x^{2} z$, $z \rightarrow u_{2}^{n} v_{2}^{5}$ so $u_{1}^{i} u_{2}^{n+1}=x^{2} u_{1}^{i} z$ with $u_{1}^{i} z \rightarrow u_{1}^{i} u_{2}^{n} v_{2}^{5}$. From Proposition 19.3 we know that $u_{1}^{m} u_{2}^{n+1} \neq 0$ and so $u_{1}^{i} u_{2}^{n+1}$ must also be nonzero.

The element $u_{2}^{n+2}$ is zero when $n \equiv 1,6,7$ or $8 \bmod 8$. Otherwise it is divisible by $x^{4}$ and by Remark 8.2 we have $x^{3} u_{1}^{4}=0$.

\section{Nonimmersions}

In this section we finish off the proofs of our nonimmersion results. We start with the first part of Theorem 1.9.

Our goal is to show that the axial map

$$
R P^{2 n} \times R P^{2^{K}-2 k-4} \longrightarrow R P^{2^{K}-2 n-2}
$$

does not exist for certain $n$ and $k$. If $n \equiv 0$ or $7 \bmod 8$,

$$
0=u^{2^{K-1}-n} \in E R(2)^{*}\left(R P^{2^{K}-2 n-2}\right) .
$$

If we show that the image of this element in $E R(2)^{*}\left(R P^{2 n} \times R P^{2^{K}-2 k-4}\right)$ is nonzero, then the axial map does not exist and $R P^{2 n}$ does not immerse in $\mathbb{R}^{2 k+2}$.

This computation is actually a coproduct because it can first be carried out for the map $R P^{\infty} \leftarrow R P^{\infty} \times R P^{\infty}$ and this last space has a Künneth isomorphism for both our theories $E R(2)^{*}(-)$ and $E(2)^{*}(-)$. As in the introduction,

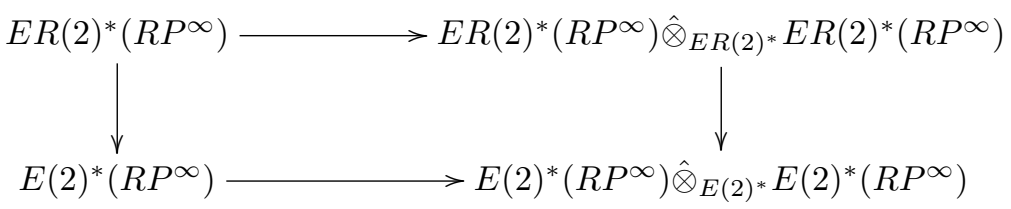

is an isomorphism from the top row to the bottom in degrees $16 *$ by Corollaries 8.3 and 17.3. The coproduct is therefore the same in both cases and comes from

$$
u \longrightarrow m^{*}(u)=u_{1}+{ }_{F} u_{2}=u_{1}+u_{2}+u_{1} u_{2} G
$$

where $G$ is a power series. We are looking at $m^{*}(u)^{2^{K-1}-n}$. If we write this out in our 2 -adic basis, it is

$$
\sum a_{k, i} \alpha^{k} u_{1}^{i} u_{2}+\sum b_{i, j} u_{1}^{i} u_{2}^{j}
$$

with $j>1$ and the $a_{k, i}$ and $b_{i, j}$ either 0 or 1 . This is the same formula for either $E R(2)^{*}(-)$ or $E(2)^{*}(-)$. The way we do this reduction is to use our algorithm, 15.3. 
Our algorithm never lowers the sum of powers of $u_{1}$ and $u_{2}$, so $i+j \geqslant 2^{K-1}-n$ for example. The 2-adic basis is the same in both cases and the relations and the reductions are also the same.

We continue the above map to

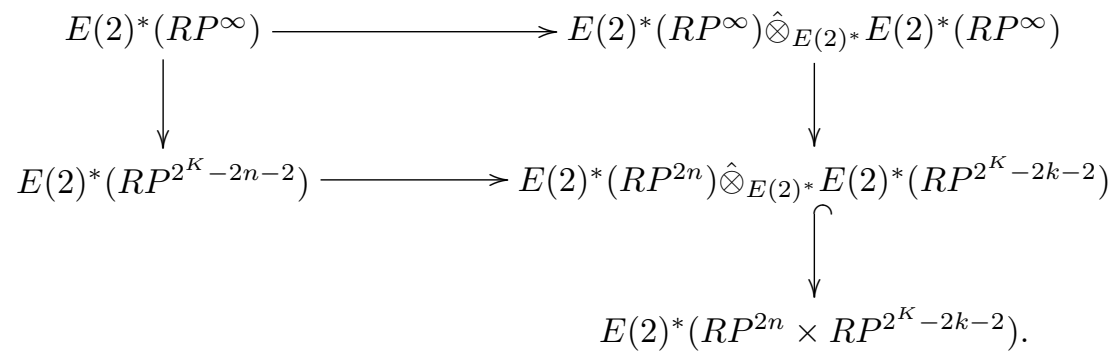

For the $E(2)^{*}(-)$ case, Don Davis, in [Dav84], showed that $0=u^{2^{K-1}-n}$ mapped to nonzero when

$$
n=m+\alpha(m)-1 \text { and } k=2 m-\alpha(m) .
$$

The top map on the right going down takes basis elements to zero or to basis elements. Since $u_{1}^{n+1}=0=u_{2}^{2^{K-1}-k}$, our coproduct reduces to

$$
\sum_{i \leqslant n} a_{k, i} \alpha^{k} u_{1}^{i} u_{2}+\sum_{\substack{i \leqslant n \\ 1<j \leqslant 2^{K-1}-k-1}} b_{i, j} u_{1}^{i} u_{2}^{j}
$$

and [Dav84] shows that this must be nonzero. We can simplify this further though. We already know that $i+j \geqslant 2^{K-1}-n$ and that $n$ is very small compared with $2^{K-1}$. Thus the first sum above, involving $\alpha^{k} u_{1}^{i} u_{2}$, is not there. The only basis elements that matter are the $u_{1}^{i} u_{2}^{j}$ with $i+j$ big, $i \leqslant n$ and $j \leqslant 2^{K-1}-k-1$. For the maximal $j=$ $2^{K-1}-k-1$, we can see, using $i+j \geqslant 2^{K-1}-n$, that $i \geqslant k+1-n$. This number is always bigger than 4 in our applications.

We now do the same thing with $E R(2)^{*}(-)$. We use the diagram, which includes our hypothetical axial map:

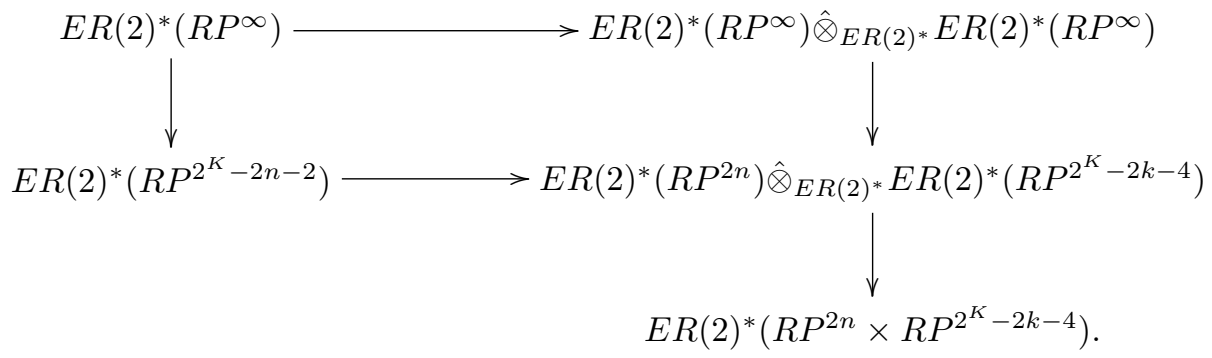

We assume that $n \equiv 0$ or $7 \bmod 8$, which gives us $u_{1}^{n+1}=0=u^{2^{K-1}-n}$, and $-k-2 \equiv$ 1, 2, 5 or $6 \bmod 8$.

Our coproduct, in either case, is contained in the $16 *$ degree part generated by $u_{1}$ and $u_{2}$ with $u_{1}^{i} u_{2}^{j}$ having big $i+j$. Furthermore, and this is important, in both cases we have $u_{1}^{n+1} u_{2}^{j}=0$ and $u_{1}^{4}{u_{2}^{2}}^{K}-k=0$. We have the same 2 -adic basis for this in both 
cases by Corollary 19.4, i.e. for

$$
E(2)^{*}\left(R P^{2 n} \times R P^{2^{K}-2 k-2}\right) \text { and } E R(2)^{*}\left(R P^{2 n} \times R P^{2^{K}-2 k-4}\right) .
$$

Consequently, our obstruction is exactly the same linear combination for $E R(2)^{*}(-)$ as it was for $E(2)^{*}(-)$ and since our obstruction is nonzero in one place it must be nonzero in our new situation.

As a result of the above discussion, Don Davis's obstructions work for us as well but with an improvement, in our special cases, of 2 . To meet our conditions we must have (from Theorem 13.4)

$$
-k-2 \equiv\{1,2,5,6\} \quad \bmod 8
$$

and, from [Dav84], $k=2 m-\alpha(m)$ and also

$$
n=m+\alpha(m)-1 \equiv\{0,7\} \bmod 8 .
$$

(from both [Dav84] and Theorem 13.4). Our result in the Introduction follows once we get our pairs $(m, \alpha(m))$ from these equations. Our first is:

$$
\begin{gathered}
-2 m+\alpha(m)-2 \equiv\{1,2,5,6\} \quad \bmod 8 ; \\
-2 m+\alpha(m) \equiv\{3,4,7,0\} \quad \bmod 8 ; \\
2 m-\alpha(m) \equiv\{5,4,1,0\} \quad \bmod 8 .
\end{gathered}
$$

The second:

$$
\begin{gathered}
m+\alpha(m)-1 \equiv\{0,7\} \quad \bmod 8 ; \\
m+\alpha(m) \equiv\{1,0\} \quad \bmod 8 .
\end{gathered}
$$

Adding the two equations we get

$$
3 m \equiv\{6,5,2,1\} \text { or }\{5,4,1,0\} \bmod 8 .
$$

Multiply by $3(\bmod 8)$ to get

$$
m \equiv\{2,7,6,3\} \text { or }\{7,4,3,0\} \bmod 8 .
$$

Substituting this into

$$
\alpha(m) \equiv-m+\{1,0\} \quad \bmod 8,
$$

we get

$$
\alpha(m)=\{7,2,3,6\} \text { or }\{1,4,5,0\} .
$$

So our result is as stated in the Theorem 1.9.

For the second part of Theorem 1.9 we begin again with the main theorem of [Dav84], for

$$
n=m+\alpha(m)-1 ; \quad k=2 m-\alpha(m) ;
$$

there does not exist an axial map

$$
R P^{2^{K}-2 k-2} \times R P^{2 n} \longrightarrow R P^{2^{K}-2 n-2},
$$

and so $R P^{2 n} \nsubseteq \mathbb{R}^{2 k}$. This is proven by using the equivalent of $E(2)^{*}(-)$ and showing that the $u^{2^{K-1}-n}=0$ on the right would have to go to a nonzero element on the left. 
That same element would prevent the existence of an axial map,

$$
R P^{2^{K}-2 k-2} \times R P^{2 n+2} \longrightarrow R P^{2^{K}-2 n-2},
$$

and likewise

$$
R P^{2^{K}-2 k-2} \times R P^{2 n+2} \longrightarrow R P^{2^{K}-2 n-4} .
$$

Furthermore, if $u^{2^{K-1}-n}$ went to nonzero then we must also have $u^{2^{K-1}-n-1}=$ 0 also going to a nonzero element. If $n+1 \equiv 7 \bmod 8$, then $u^{2^{K-1}-n-1}=0$ for $E R(2)^{*}\left(R P^{2^{K}-2 n-4}\right)$ and, if $-k-2 \equiv\{1,2,5,6\} \bmod 8$ this must factor through the $E R(2)^{*}(-)$ cohomology of

$$
R P^{2^{K}-2 k-4} \times R P^{2 n+2} \longrightarrow R P^{2^{K}-2 n-4}
$$

as above and we have that $R P^{2 n+2} \nsubseteq \mathbb{R}^{2 k+2}$, or,

$$
R P^{2(m+\alpha(m))} \nsubseteq \mathbb{R}^{2(2 m-\alpha(m)+1)} .
$$

We have to untangle some equations to get our $(m, \alpha(m))$ pairs for this. We have

$$
n+1=m+\alpha(m) \equiv 7 \bmod 8
$$

and

$$
-k-2 \equiv-2 m+\alpha(m)-2 \equiv\{1,2,5,6\} \quad \bmod 8 .
$$

The equation for $k$ is the same as before so we have

$$
2 m-\alpha(m) \equiv\{5,4,1,0\} \quad \bmod 8 .
$$

The equation for $n$ gives

$$
m+\alpha(m) \equiv 7 \quad \bmod 8
$$

Adding, we have

$$
3 m \equiv\{4,3,0,7\} \quad \bmod 8 .
$$

Multiply by 3 to get

$$
m \equiv\{4,1,0,5\} \quad \bmod 8
$$

Substituting into

$$
\alpha(m) \equiv-m+7 \bmod 8,
$$

we get

$$
\alpha(m)=\{3,6,7,2\}
$$

and our pairs are as in our Theorem 1.9.

Remark 20.1. Don Davis does his work with the theory $B P\langle 2\rangle^{*}(-)$ with $B P\langle 2\rangle^{*} \simeq$ $\mathbb{Z}_{(2)}\left[v_{1}, v_{2}\right]$. For these spaces there is no $v_{2}$ torsion so when $v_{2}$ is inverted to create $E(2)^{*}(-)$, everything injects. The normal $E(2)$ is 6 -periodic but we can consider it 48 periodic just as well, it doesn't change anything. Davis does all of his computations with the standard 2-dimensional class, $x_{2}$, but the computations all hold if this is adjusted by a unit so we can use our $u$ in degree -16 . 


\section{The Atiyah-Hirzebruch spectral sequence approach}

The original computation of $E R(2)^{*}\left(R P^{2 n}\right)$ was carried out using the AtiyahHirzebruch spectral sequence and we give a brief description of how that was done here. To begin we use the long exact sequence:

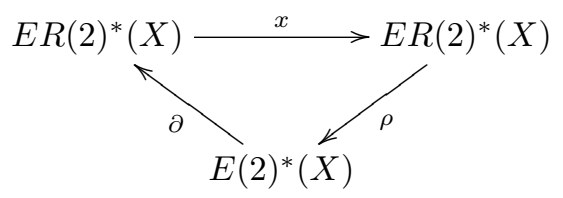

for $X=R P^{16}$ and $X=R P^{\infty}$. Since we know $E(2)^{*}\left(R P^{16}\right)$, we can just look at the Atiyah-Hirzebruch spectral sequence for $E R(2)^{*}\left(R P^{16}\right)$ and see that what is there in the $E^{2}$-term for $E R(2)^{16 *}\left(R P^{16}\right)$ must map isomorphically to $E(2)^{16 *}\left(R P^{16}\right)$ and so must also be $E^{\infty}$ and cannot have any differentials entering or leaving. Using this isomorphism and the fact that $E(2)^{*}\left(R P^{16}\right)$ is even degree, the long exact sequence gives us $E R(2)^{16 *+1}\left(R P^{16}\right)=0$ as it is trapped in:

$0 \simeq E(2)^{16 *-1}\left(R P^{16}\right) \rightarrow E R(2)^{16 *+17}\left(R P^{16}\right) \rightarrow E R(2)^{16 *}\left(R P^{16}\right) \simeq E(2)^{16 *}\left(R P^{16}\right)$.

It then follows that $E R(2)^{16 *+2}\left(R P^{16}\right)=0$, from

$$
\begin{gathered}
E R(2)^{16 *}\left(R P^{16}\right) \simeq E(2)^{16 *}\left(R P^{16}\right) \longrightarrow \\
E R(2)^{16 *+18}\left(R P^{16}\right) \longrightarrow E R(2)^{16 *+1}\left(R P^{16}\right)=0 .
\end{gathered}
$$

We get one more, i.e. $E R(2)^{16 *+3}\left(R P^{16}\right)=0$ from

$$
0 \simeq E(2)^{16 *-15}\left(R P^{16}\right) \longrightarrow E R(2)^{16 *+3}\left(R P^{16}\right) \longrightarrow E R(2)^{16 *+2}\left(R P^{16}\right)=0 .
$$

In order for the Atiyah-Hirzebruch spectral sequence for $E R(2)^{*}\left(R P^{16}\right)$ to end up with zero in these degrees we must have differentials, none of which can start (or end) on $E R(2)^{16 *}\left(R P^{16}\right)$. There is only one way for this to happen and it shows us what the $d^{r}$ are for $r=2,3,4,5,6$ and 7 . These differentials then work for all $E R(2)^{*}\left(R P^{2 n}\right)$. Elements can be identified using $E R(2)^{*}\left(R P^{\infty}\right)$ and the map to $E(2)^{*}\left(R P^{\infty}\right)$.

This works quite well but breaks down when attempting products.

\section{References}

[BDM02] R. R. Bruner, D. M. Davis, and M. Mahowald, Nonimmersions of real projective spaces implied by tmf, Recent Progress in Homotopy Theory: Proceedings of a Conference on Recent Progress in Homotopy Theory (D. Davis, J. Morava, G. Nishida, W. S. Wilson, and N. Yagita, eds.), March 17-27, 2000, Johns Hopkins University, Baltimore, MD., 45-68, Contemp. Math. 293, Providence, RI, Amer. Math. Soc., 2002.

[Dav] D. Davis, Table of immersions and embeddings of real projective spaces, http://www.lehigh.edu/ dmd1/immtable.

[Dav84] D. M. Davis, A strong nonimmersion theorem for real projective spaces, Ann. of Math, 120 (1984), 517-528. 
[GW] J. González and W.S. Wilson, The BP-theory of two-fold products of projective spaces, Homology, Homotopy Appl. 10(3) (2008), 181-192.

[HK01] P. Hu and I. Kriz, Real-oriented homotopy theory and an analogue of the Adams-Novikov spectral sequence, Topology 40 (2001), 317-399.

[Hu01] P. Hu, The Ext $t^{0}$-term of the real-oriented Adams-Novikov spectral sequence, Homotopy Methods in Algebraic Topology, 141-153, Contemp. Math. 271, Providence, RI, Providence, Rhode Island, Amer. Amer. Math. Soc., 2001.

[Jam63] I.M. James, On the immersion problem for real projective spaces, Bull. Amer. Math. Soc. 69 (1963), 231-238.

[JW85] D. C. Johnson and W. S. Wilson, The Brown-Peterson homology of elementary p-groups, Amer. J. Math. 107 (1985), 427-454.

[KW07a] N. Kitchloo and W.S. Wilson, On fibrations related to real spectra, Proceedings of the Nishida Fest (Kinosaki 2003) (M. Ando, N. Minami, J. Morava, and W.S. Wilson, eds.), Geometry \& Topology Monographs, vol. 10, 237-244, 2007.

[KW07b] N. Kitchloo and W.S. Wilson, On the Hopf ring for ER(n), Topology Appl. 154 (2007), 1608-1640.

[Lan70] P. S. Landweber, Coherence, flatness and cobordism of classifying spaces, Proceedings of Advanced Study Institute on Algebraic Topology, 256-269, Aarhus, 1970.

[LMS86] L. G. Lewis, J. P. May, and M. Steinberger, Equivariant Stable Homotopy Theory, Springer Lecture Notes in Math. vol. 1213, Springer-Verlag, New York, 1986.

[Mas54] W.S. Massey, Products in exact couples, Ann. of Math. 59(3) (1954), 558-569.

Nitu Kitchloo nitu@math.ucsd.edu

Department of Mathematics, University of California, San Diego (UCSD), La Jolla, CA 92093-0112, USA

W. Stephen Wilson wsw@math.jhu.edu

Department of Mathematics, Johns Hopkins University, Baltimore, MD 21218, USA 